\title{
Hankel determinants for the Fibonacci word and Padé approximation
}

\author{
by \\ Teturo Kamae (Osaka), Jun-IChi Tamura (Tokyo) \\ and ZHI-YING WEN (Beijing)
}

1. Introduction. The aim of this paper is to give a concrete and interesting example of the Padé approximation theory as well as to develop the general theory so as to find a quantitative relation between the Hankel determinant and the Padé pair. Our example is the formal power series related to the Fibonacci word.

The Fibonacci word $\varepsilon(a, b)$ on an alphabet $\{a, b\}$ is the infinite sequence

$$
\begin{aligned}
\varepsilon(a, b) & =\widehat{\varepsilon}_{0} \widehat{\varepsilon}_{1} \ldots \widehat{\varepsilon}_{n} \ldots \\
& :=\text { abaababaabaab } \ldots \quad\left(\widehat{\varepsilon}_{n} \in\{a, b\}\right),
\end{aligned}
$$

which is the fixed point of the substitution

$$
\sigma: \quad a \rightarrow a b, \quad b \rightarrow a .
$$

The Hankel determinants for an infinite word (or sequence) $\varphi=\varphi_{0} \varphi_{1} \ldots$ $\left(\varphi_{n} \in \mathbb{K}\right)$ over a field $\mathbb{K}$ are

(3) $\quad H_{n, m}(\varphi):=\operatorname{det}\left(\varphi_{n+i+j}\right)_{0 \leq i, j \leq m-1} \quad(n=0,1, \ldots ; m=1,2, \ldots)$.

It is known [2] that the Hankel determinants play an important role in the theory of Padé approximation for the formal Laurent series

$$
\varphi(z)=\sum_{k=0}^{\infty} \varphi_{k} z^{-k+h} .
$$

Let $\mathbb{K}\left(\left(z^{-1}\right)\right)$ be the set of formal Laurent series $\varphi$ as above of $z$ with coefficients in $\mathbb{K}$ and $h \in \mathbb{Z}$ providing a nonarchimedean norm $\|\varphi\|:=\exp \left(-k_{0}+h\right)$ with $k_{0}=\inf \left\{k: \varphi_{k} \neq 0\right\}$. Let $\varphi$ be as above with $h=-1$. We say that a

1991 Mathematics Subject Classification: 41A21, 11B39.

This research was partially supported by the Hatori Project of the Mathematical Society of Japan. 
pair $(P, Q) \in \mathbb{K}[z]^{2}$ of polynomials of $z$ over $\mathbb{K}$ is a Padé pair of order $m$ for $\varphi$ if

$$
\|Q \varphi-P\|<\exp (-m), \quad Q \neq 0, \quad \operatorname{deg} Q \leq m .
$$

A Padé pair $(P, Q)$ of order $m$ for $\varphi$ always exists and the rational function $P / Q \in \mathbb{K}(z)$ is uniquely determined for each $m=0,1, \ldots$ The element $P / Q \in \mathbb{K}(z)$ with $P, Q$ satisfying (5) is called the $m$ th diagonal Padé approximation for $\varphi$. A number $m$ is called a normal index if (5) implies $\operatorname{deg} Q=m$. Note that $P / Q$ is irreducible if $m$ is a normal index, although it can be reducible for a general $m$. A normal Padé pair $(P, Q)$, i.e., $\operatorname{deg} Q$ is a normal index, is said to be normalized if the leading coefficient of $Q$ is equal to 1 . It is a classical result that $m$ is a normal index for $\varphi$ if and only if the Hankel determinant $\operatorname{det}\left(\varphi_{i+j}\right)_{0 \leq i, j \leq m-1}$ is nonzero. Note that 0 is always a normal index and the determinant for the empty matrix is considered to be 1 , so that the above statement remains valid for $m=0$.

We succeed in obtaining a quantitative relation between the Hankel determinant and the normalized Padé pair. Namely,

$$
\operatorname{det}\left(\varphi_{i+j}\right)_{0 \leq i, j \leq m-1}=(-1)^{[m / 2]} \prod_{z ; Q(z)=0} P(z)
$$

for any normal index $m$ with the normalized Padé pair $(P, Q)$, where $\prod_{z ; Q(z)=0}$ indicates a product taken over all zeros $z$ of $Q$ with their multiplicity (Theorem 6).

We are specially interested in the Padé approximation theory applied to the Fibonacci words $\varepsilon:=\varepsilon(1,0)$ and $\bar{\varepsilon}:=\varepsilon(0,1)$, where 0,1 are considered as elements in the field $\mathbb{Q}$, since we have the following remark.

REMARK 1. Let $M$ be a matrix of size $m \times m$ with entries consisting of two independent variables $a$ and $b$. Then $\operatorname{det} M=(a-b)^{m-1}\left(p a+(-1)^{m-1} q b\right)$, where $p$ and $q$ are integers defined by

$$
p=\left.\operatorname{det} M\right|_{a=1, b=0}, \quad q=\left.\operatorname{det} M\right|_{a=0, b=1} .
$$

Proof. Subtracting the first column vector from all the other column vectors of $M$, we see that $\operatorname{det} M$ is divisible by $(a-b)^{m-1}$ as a polynomial in $\mathbb{Z}[a, b]$. Hence, $\operatorname{det} M=(a-b)^{m-1}(x a+y b)$ for integers $x, y$. Setting $(a, b)=(1,0),(0,1)$, we get the assertion.

In Section 2, we study the structure of the Fibonacci word, in particular, its repetition property. The notion of singular words introduced in Z.-X. Wen and Z.-Y. Wen [5] plays an important role.

In Section 3, we give the value of the Hankel determinants $H_{n, m}(\varepsilon)$ and $H_{n, m}(\bar{\varepsilon})$ for the Fibonacci words in some closed forms. It is a rare case where the Hankel determinants are determined completely. Another such case is for the Thue-Morse sequence $\varphi$ consisting of 0 and 1, where the Hankel 
determinants $H_{m, n}(\varphi)$ modulo 2 are obtained, and the function $H_{m, n}(\varphi)$ of $(m, n)$ is proved to be 2-dimensionally automatic (see [1]).

In Section 4, we consider the self-similar property of the values $H_{n, m}(\varepsilon)$ and $H_{n, m}(\bar{\varepsilon})$ for the Fibonacci words. The quarter plane $\{(n, m): n \geq 0$, $m \geq 1\}$ is tiled by 3 kinds of tiles with the values $H_{n, m}(\varepsilon)$ and $H_{n, m}(\bar{\varepsilon})$ on it with various scales.

In Section 5, we develop a general theory of Padé approximation. We also obtain the admissible continued fraction expansion of $\varphi_{\varepsilon}$ and $\varphi_{\bar{\varepsilon}}$, the formal Laurent series (4) with $h=-1$ for the sequences $\varepsilon$ and $\bar{\varepsilon}$, and determine all the convergents $p_{k} / q_{k}$ of the continued fractions. It is known in general that the set of the convergents $p_{k} / q_{k}$ for $\varphi$ is the set of diagonal Padé approximations and the set of degrees of $q_{k}$ 's in $z$ coincides with the set of normal indices for $\varphi$.

2. Structure of the Fibonacci word. In what follows, $\sigma$ denotes the substitution defined by (2), and

$$
\widehat{\varepsilon}=\widehat{\varepsilon}_{0} \widehat{\varepsilon}_{1} \ldots \widehat{\varepsilon}_{n} \ldots \quad\left(\widehat{\varepsilon}_{n} \in\{a, b\}\right)
$$

is the (infinite) Fibonacci word (1). A finite word over $\{a, b\}$ is sometimes considered to be an element of the free group generated by $a$ and $b$ with inverses $a^{-1}$ and $b^{-1}$. For $n=0,1, \ldots$, we define the $n$th Fibonacci word $F_{n}$ and the $n$th singular word $W_{n}$ as follows:

$$
F_{n}:=\sigma^{n}(a)=\sigma^{n+1}(b), \quad W_{n}:=\beta_{n} F_{n} \alpha_{n}^{-1},
$$

where we put

$$
\alpha_{n}=\beta_{m}= \begin{cases}a & (n \text { even }, m \text { odd }), \\ b & (n \text { odd }, m \text { even }),\end{cases}
$$

and we define $W_{-2}$ to be the empty word and $W_{-1}:=a$ for convenience. Let $\left(f_{n} ; n \in \mathbb{Z}\right)$ be the Fibonacci sequence:

$$
f_{n+2}=f_{n+1}+f_{n} \quad(n \in \mathbb{Z}), \quad f_{-1}=f_{0}=1 .
$$

Then $\left|F_{n}\right|=\left|W_{n}\right|=f_{n}(n \geq 0)$, where $|\xi|$ denotes the length of a finite word $\xi$.

For a finite word $\xi=\xi_{0} \xi_{1} \ldots \xi_{n-1}$ and a finite or infinite word $\eta=$ $\eta_{0} \eta_{1} \ldots$ over an alphabet, we denote

$$
\xi \prec_{k} \eta
$$

if $\xi=\eta_{k} \eta_{k+1} \ldots \eta_{k+n-1}$. We simply write

$$
\xi \prec \eta
$$

and say that $\xi$ is a subword of $\eta$ if $\xi \prec_{k} \eta$ for some $k$. For a finite word $\xi=\xi_{0} \xi_{1} \ldots \xi_{n-1}$ and $i$ with $0 \leq i<n$, we denote the $i$ th cyclic permutation 
of $\xi$ by $C_{i}(\xi):=\xi_{i} \xi_{i+1} \ldots \xi_{n-1} \xi_{0} \xi_{1} \ldots \xi_{i-1}$. We also define $C_{i}(\xi):=C_{i^{\prime}}(\xi)$ with $i^{\prime}:=i-n[i / n]$ for any $i \in \mathbb{Z}$.

In this section, we study the structure of the Fibonacci word $\widehat{\varepsilon}$ and discuss the repetition property. The following two lemmas were obtained by Z.-X. Wen and Z.-Y. Wen [5] and we omit the proofs.

LEMMA 1. We have the following statements:

(1) $\widehat{\varepsilon}=F_{n} F_{n-1} F_{n} F_{n+1} F_{n+2} \ldots(n \geq 1)$,

(2) $F_{n}=F_{n-1} F_{n-2}=F_{n-2} F_{n-1} \beta_{n}^{-1} \alpha_{n}^{-1} \beta_{n} \alpha_{n}(n \geq 2)$,

(3) $F_{n} F_{n} \prec \widehat{\varepsilon}(n \geq 3)$,

(4) $\widehat{\varepsilon}=W_{-1} W_{0} W_{1} W_{2} W_{3} \ldots$,

(5) $W_{n}=W_{n-2} W_{n-3} W_{n-2}(n \geq 1)$,

(6) $W_{n}$ is a palindrome, that is, $W_{n}$ stays invariant under reading the letters from the end $(n \geq-2)$,

(7) $C_{i}\left(F_{n}\right) \prec \widehat{\varepsilon}\left(n \geq 0,0 \leq i<f_{n}\right)$,

(8) $C_{i}\left(F_{n}\right) \neq C_{j}\left(F_{n}\right)$ for any $i \neq j$, moreover, they are different already before their last places $\left(n \geq 1,0 \leq i<f_{n}\right)$,

(9) $W_{n} \neq C_{i}\left(F_{n}\right)\left(n \geq 0,0 \leq i<f_{n}\right)$,

(10) $\xi \prec \widehat{\varepsilon}$ and $|\xi|=f_{n}$ imply that either $\xi=C_{i}\left(F_{n}\right)$ for some $i$ with $0 \leq i<f_{n}$ or $\xi=W_{n}(n \geq 0)$.

Lemma 2. For any $k \geq-1$, we have the decomposition of $\widehat{\varepsilon}$ as follows:

$$
\widehat{\varepsilon}=\left(W_{-1} W_{0} \ldots W_{k-1}\right) W_{k} \gamma_{0} W_{k} \gamma_{1} \ldots W_{k} \gamma_{n} \ldots,
$$

where all the occurrences of $W_{k}$ in $\widehat{\varepsilon}$ are picked up and $\gamma_{n}$ is either $W_{k+1}$ or $W_{k-1}$ corresponding to $\widehat{\varepsilon}_{n}$ is a or $b$, respectively. That is, any two different occurrences of $W_{k}$ do not overlap and are separated by $W_{k+1}$ or $W_{k-1}$.

We introduce another method to discuss the repetition property of $\widehat{\varepsilon}$. Let $\mathbb{N}$ be the set of nonnegative integers. For $n \in \mathbb{N}$, let

$$
\begin{aligned}
& n=\sum_{i=0}^{\infty} \tau_{i}(n) f_{i}, \\
& \tau_{i}(n) \in\{0,1\} \quad \text { and } \tau_{i}(n) \tau_{i+1}(n)=0 \quad(i \in \mathbb{N})
\end{aligned}
$$

be the regular expression of $n$ in the Fibonacci base due to Zeckendorf. For $m, n \in \mathbb{N}$ and a positive integer $k$, we define

$$
m \equiv_{k} n
$$

if $\tau_{i}(m)=\tau_{i}(n)$ for all $i<k$.

Lemma 3. We have $\widehat{\varepsilon}_{n}=a$ if and only if $\tau_{0}(n)=0$.

Pr o of. We use induction on $n$. The lemma holds for $n=0,1,2$. Assume that it holds for any $n \in \mathbb{N}$ with $n<f_{k}$ for some $k \geq 2$. Take any $n \in \mathbb{N}$ with $f_{k} \leq n<f_{k+1}$. Then, since $0 \leq n-f_{k}<f_{k-1}$, we have 


$$
n=\sum_{i=0}^{k-1} \tau_{i}\left(n-f_{k}\right) f_{i}+f_{k}
$$

which gives the regular expression if $\tau_{k-1}\left(n-f_{k}\right)=0$. If $\tau_{k-1}\left(n-f_{k}\right)=1$, then we have the regular expression $n=\sum_{i=0}^{k-2} \tau_{i}\left(n-f_{k}\right) f_{i}+f_{k+1}$. In any case, we have $\tau_{0}(n)=\tau_{0}\left(n-f_{k}\right)$. On the other hand, since $\widehat{\varepsilon}$ starts with $F_{k} F_{k-1}$ by Lemma 1 , we have $\widehat{\varepsilon}_{n}=\widehat{\varepsilon}_{n-f_{k}}$. Hence, $\widehat{\varepsilon}_{n}=a$ if and only if $\tau_{0}(n)=0$ by the induction hypothesis. Thus, we have the assertion for any $n<f_{k+1}$, and by induction, we complete the proof.

Lemma 4. Let $n=\sum_{i=0}^{\infty} n_{i} f_{i}$ with $n_{i} \in\{0,1\}(i \in \mathbb{N})$. Assume that $n_{i} n_{i+1}=0$ for $0 \leq i<k$. Then $n_{i}=\tau_{i}(n)$ for $0 \leq i<k$.

Proof. If there exists $i \in \mathbb{N}$ such that $n_{i} n_{i+1}=1$, let $i_{0}$ be the maximum such $i$. Take the maximum $j$ such that $n_{i_{0}+1}=n_{i_{0}+3}=n_{i_{0}+5}=\ldots=n_{j}$ $=1$. Then, replacing $f_{i_{0}}+f_{i_{0}+1}+f_{i_{0}+3}+f_{i_{0}+5}+\ldots+f_{j}$ by $f_{j+1}$, we have a new expression of $n$ :

$$
n=\sum_{i=0}^{\infty} n_{i}^{\prime} f_{i}:=\sum_{i=0}^{i_{0}-1} n_{i} f_{i}+f_{j+1}+\sum_{i=j+3}^{\infty} n_{i} f_{i} .
$$

This new expression is unchanged at the indices less than $k$, and is either regular or has a smaller maximum index $i$ with $n_{i}^{\prime} n_{i+1}^{\prime}=1$. By continuing this procedure, we finally get the regular expression of $n$, which does not differ from the original expression at the indices less than $k$. Thus, $n_{i}=\tau_{i}(n)$ for any $0 \leq i<k$.

Lemma 5. For any $n \in \mathbb{N}$ and $k \geq 0, \tau_{0}\left(n+f_{k}\right) \neq \tau_{0}(n)$ if and only if either $n \equiv_{k+2} f_{k+1}-2$ or $n \equiv_{k+2} f_{k+1}-1$. Moreover,

$$
\widehat{\varepsilon}_{n+f_{k}}-\widehat{\varepsilon}_{n}= \begin{cases}(-1)^{k-1}(a-b) & \left(n \equiv_{k+2} f_{k+1}-2\right), \\ (-1)^{k}(a-b) & \left(n \equiv_{k+2} f_{k+1}-1\right),\end{cases}
$$

where $a$ and $b$ are considered as independent variables.

P r o of. If $k=0$, we can verify the statement by a direct calculation.

Assume that $k \geq 1$ and $\tau_{k}(n)=0$. Then

$$
n+f_{k}=\sum_{i=0}^{k-1} \tau_{i}(n) f_{i}+f_{k}+\sum_{i=k+1}^{\infty} \tau_{i}(n) f_{i} .
$$

By Lemma 4, we have $\tau_{0}\left(n+f_{k}\right)=\tau_{0}(n)$ if $k \geq 2$ or if $k=1$ and $\tau_{0}(n)=0$. In the case where $k=1, \tau_{0}(n)=1$ and $\tau_{2}(n)=0$, since

$$
n+f_{k}=1+2+\sum_{i=3}^{\infty} \tau_{i}(n) f_{i}=f_{2}+\sum_{i=3}^{\infty} \tau_{i}(n) f_{i},
$$

we have $\tau_{0}\left(n+f_{k}\right)=0$ by Lemma 4 . On the other hand, in the case where $k=1, \tau_{0}(n)=1$ and $\tau_{2}(n)=1$, since 


$$
n+f_{k}=1+2+3+\sum_{i=4}^{\infty} \tau_{i}(n) f_{i}=f_{0}+f_{3}+\sum_{i=4}^{\infty} \tau_{i}(n) f_{i},
$$

we have $\tau_{0}\left(n+f_{k}\right)=1$ by Lemma 4 .

Thus, in the case where $k \geq 1$ and $\tau_{k}(n)=0, \tau_{0}\left(n+f_{k}\right) \neq \tau_{0}(n)$ if and only if $k=1, \tau_{0}(n)=1$ and $\tau_{2}(n)=0$, or equivalently, if and only if $n \equiv_{k+2} f_{k+1}-2$ with $k=1$. Note that $n \equiv_{k+1} f_{k+1}-1$ with $k=1$ contradicts $\tau_{k}(n)=0$.

Now assume that $k \geq 1$ and $\tau_{k}(n)=1$. Take the minimum $j \geq 0$ such that $\tau_{k}(n)=\tau_{k-2}(n)=\tau_{k-4}(n)=\ldots=\tau_{j}(n)=1$. Then since $2 f_{i}=$ $f_{i+1}+f_{i-2}$ for any $i \in \mathbb{N}$, we have

$$
\begin{aligned}
n+f_{k}= & \sum_{i=0}^{j-3} \tau_{i}(n) f_{i}+f_{j-2} \\
& +f_{j+1}+f_{j+3}+f_{j+5}+\ldots+f_{k+1}+\sum_{i=k+2}^{\infty} \tau_{i}(n) f_{i},
\end{aligned}
$$

where the first term on the right-hand side vanishes if $j=0,1,2$. Hence by Lemma $4, \tau_{0}\left(n+f_{k}\right)=\tau_{0}(n)$ if $j \geq 4$.

In the case where $j=3, \tau_{0}\left(n+f_{k}\right)=\tau_{0}(n)$ holds if $\tau_{0}(n)=0$ by $(14)$ and Lemma 4. If $\tau_{0}(n)=1$, then by (14) and Lemma $4, \tau_{0}\left(n+f_{k}\right)=0$. Thus, for $j=3, \tau_{0}\left(n+f_{k}\right) \neq \tau_{0}(n)$ if and only if $\tau_{0}(n)=1$.

If $j=2$, then by the assumption on $j$, we have $\tau_{0}(n)=0$. On the other hand, since $f_{0}=1$, by (14) and Lemma 4, we have $\tau_{0}\left(n+f_{k}\right)=1$. Thus, $\tau_{0}\left(n+f_{k}\right) \neq \tau_{0}(n)$.

If $j=1$, then $\tau_{0}(n)=0$ since $\tau_{1}(n)=1$ by the assumption on $j$. On the other hand, since $f_{-1}=1$, we have $\tau_{0}\left(n+f_{k}\right)=1$ by (14) and Lemma 4 . Thus, $\tau_{0}\left(n+f_{k}\right) \neq \tau_{0}(n)$.

If $j=0$, then by the assumption on $j, \tau_{0}(n)=1$. On the other hand, since $f_{-2}=0$, we have $\tau_{0}\left(n+f_{k}\right)=0$ by (14) and Lemma 4 . Thus, $\tau_{0}\left(n+f_{k}\right) \neq$ $\tau_{0}(n)$.

By combining all the results as above, we get the first part.

The second part follows from Lemma 3 and the fact that for any $k \geq 0$,

$$
f_{k+1}-1=f_{k}+f_{k-2}+\ldots+f_{i}
$$

with $i=0$ if $k$ is even and $i=1$ if $k$ is odd. Hence,

$$
\tau_{0}\left(f_{k+1}-1\right)=\tau_{0}\left(f_{h+1}-2\right)= \begin{cases}a & (k \text { odd }, h \text { even }), \\ b & (k \text { even, } h \text { odd }) .\end{cases}
$$

Lemma 6. For any $k \geq 0, W_{k} \prec_{n} \widehat{\varepsilon}$ if and only if $n \equiv_{k+2} f_{k+1}-1$.

Proof. By Lemma 2, the smallest $n \in \mathbb{N}$ such that $W_{k} \prec_{n} \widehat{\varepsilon}$ is

$$
f_{-1}+f_{0}+f_{1}+\ldots+f_{k-1}=f_{k+1}-1,
$$


which is the smallest $n \in \mathbb{N}$ such that $n \equiv_{k+2} f_{k+1}-1$. Let $n_{0}:=f_{k+1}-1$. Then the regular expression of $n_{0}$ is

$$
n_{0}=f_{k}+f_{k-2}+f_{k-4}+\ldots+f_{d},
$$

where $d=\left(1-(-1)^{k}\right) / 2$. The next $n$ with $n \equiv_{k+2} n_{0}$ is clearly

$$
n=f_{k+2}+f_{k}+f_{k-2}+\ldots+f_{d},
$$

which is, by Lemma 2 , the next $n$ such that $W_{k} \prec_{n} \widehat{\varepsilon}$ since $f_{k}+f_{k+1}=f_{k+2}$.

For $i=1,2, \ldots$, let

$$
n_{i}=n_{0}+\sum_{j=0}^{\infty} \tau_{j}(i) f_{k+2+j} .
$$

Then it is easy to see that $n_{i}$ is the $i$ th $n$ after $n_{0}$ such that $n \equiv_{k+2} f_{k+1}-1$. We prove by induction on $i$ that $n_{i}$ is the $i$ th $n$ after $n_{0}$ such that $W_{k} \prec_{n} \widehat{\varepsilon}$. Assume that it is so for $i$. Then by Lemma $4, W_{k} \gamma_{i} W_{k} \prec_{n_{i}} \widehat{\varepsilon}$. Hence, the next $n$ after $n_{i}$ such that $W_{k} \prec_{n} \widehat{\varepsilon}$ is $n_{i}+f_{k}+\left|\gamma_{i}\right|$. Thus, we have

$$
\begin{aligned}
n_{i}+f_{k}+\left|\gamma_{i}\right| & =n_{i}+f_{k}+f_{k+1} 1_{\widehat{\varepsilon}_{i}=a}+f_{k-1} 1_{\widehat{\varepsilon}_{i}=b} \\
& =n_{i}+f_{k+2} 1_{\tau_{0}(i)=0}+f_{k+1} 1_{\tau_{0}(i)=1}=n_{i+1},
\end{aligned}
$$

which completes the proof.

Lemma 7. Let $k \geq 0$ and $n, i \in \mathbb{N}$ satisfy $n \equiv_{k+1} i$.

(1) If $0 \leq i<f_{k}$, then $\tau_{0}(n+j)=\tau_{0}(i+j)$ for any $j=0,1, \ldots$, $f_{k+2}-i-3$.

(2) If $f_{k} \leq i<f_{k+1}$, then $\tau_{0}(n+j)=\tau_{0}(i+j)$ for any $j=0,1, \ldots$, $f_{k+3}-i-3$.

Pr o of. (1) We prove the lemma by induction on $k$. The assertion holds for $k=0$. Let $k \geq 1$ and assume that the assertion is valid for $k-1$. For $j=0,1, \ldots, f_{k}-i$, we have $n+j \equiv_{k} i+j$ and hence, $\tau_{0}(n+j)=\tau_{0}(i+j)$. Let $j_{0}=f_{k}-i$. Then, since $n+j_{0} \equiv_{k} i+j_{0} \equiv_{k} 0$, we have $\tau_{0}\left(n+j_{0}+\right.$ $j)=\tau_{0}\left(i+j_{0}+j\right)=\tau_{0}(j)$ for any $j=0,1, \ldots, f_{k+1}-3$ by the induction hypothesis. Thus, $\tau_{0}(n+j)=\tau_{0}(i+j)$ for any $j=0,1, \ldots, f_{k+2}-i-3$. This proves (1).

(2) In this case, $\tau_{k+1}(n)=0$. Hence, $n \equiv_{k+2} i$. Therefore, we can apply (1) with $k+1$ for $k$. Thus, we get (2).

Let $n, m, i \in \mathbb{N}$ with $m \geq 2$ and $0<i<m$. We call $n$ an $(m, i)$-shift invariant place in $\widehat{\varepsilon}$ if

$$
\widehat{\varepsilon}_{n} \widehat{\varepsilon}_{n+1} \ldots \widehat{\varepsilon}_{n+m-1}=\widehat{\varepsilon}_{n+i} \widehat{\varepsilon}_{n+i+1} \ldots \widehat{\varepsilon}_{n+i+m-1} .
$$

We call $n$ an $m$-repetitive place in $\widehat{\varepsilon}$ if there exist $i, j \in \mathbb{N}$ with $i>0$ and $i+j<m$ such that $n+j$ is an $(m, i)$-shift invariant place in $\widehat{\varepsilon}$. Let $\mathcal{R}_{m}$ be the set of $m$-repetitive places in $\widehat{\varepsilon}$. 
Lemma 8. (1) Let $n \equiv_{k+1} 0$ for some $k \geq 1$. Then $n$ is an $\left(f_{k+1}-2, f_{k}\right)$ shift invariant place in $\widehat{\varepsilon}$.

(2) Let $n \equiv_{k+1} f_{k}$ for some $k \geq 2$. Then $n$ is an $\left(f_{k+1}-2, f_{k-1}\right)$-shift invariant place in $\widehat{\varepsilon}$.

Proof. (1) Since the least $i \geq n$ such that either $i \equiv_{k+2} f_{k+1}-1$ or $i \equiv_{k+2} f_{k+1}-2$ is not less than $n+f_{k+1}-2$, by Lemma 5 , we have

$$
\widehat{\varepsilon}_{n} \widehat{\varepsilon}_{n+1} \ldots \widehat{\varepsilon}_{n+f_{k+1}-3}=\widehat{\varepsilon}_{n+f_{k}} \widehat{\varepsilon}_{n+f_{k}+1} \ldots \widehat{\varepsilon}_{n+f_{k}+f_{k+1}-3} .
$$

(2) Since the minimum $i \geq n$ such that either $i \equiv_{k+1} f_{k}-1$ or $i \equiv_{k+1}$ $f_{k}-2$ is $n+f_{k+1}-2$, by Lemma 5 , we have

$$
\widehat{\varepsilon}_{n} \widehat{\varepsilon}_{n+1} \ldots \widehat{\varepsilon}_{n+f_{k+1}-3}=\widehat{\varepsilon}_{n+f_{k-1}} \widehat{\varepsilon}_{n+f_{k-1}+1} \ldots \widehat{\varepsilon}_{n+f_{k-1}+f_{k+1}-3} \text {. }
$$

THEOREM 1. The pair $(n, m)$ of nonnegative integers satisfies $n \in \mathcal{R}_{m}$ if one of the following two conditions holds:

(1) $f_{k}+1 \leq m \leq f_{k+1}-2, n-i \equiv_{k+1} 0$ and $i \leq n$ for some $k \geq 1$ and $i \in \mathbb{Z}$ with $f_{k}+1 \leq m+i \leq f_{k+1}-2$.

(2) $f_{k-1}+1 \leq m \leq f_{k+1}-2, i \leq n$ and $n-i \equiv_{k+1} f_{k}$ for some $k \geq 2$ and $i \in \mathbb{Z}$ with $f_{k-1}+1 \leq m+i \leq f_{k+1}-2$.

REMARK 2. The "if and only if" statement actually holds in Theorem 1 in place of "if" since we will prove later that $H_{n, m} \neq 0$ if none of the conditions (1) and (2) hold.

Proof (of Theorem 1). Assume (1) and $i \geq 0$. By Lemma 8(1), $n-i$ is an $\left(f_{k+1}-2, f_{k}\right)$-shift invariant place. Then $n$ is an $\left(m, f_{k}\right)$-shift invariant place since $i+m \leq f_{k+1}-2$. Thus, $n \in \mathcal{R}_{m}$ as $f_{k}<m$.

Assume (1) and $i<0$. Then, since $n-i$ is an $\left(f_{k+1}-2, f_{k}\right)$-shift invariant place and $m \leq f_{k+2}-2$, it is an $\left(m, f_{k}\right)$-shift invariant place. Moreover, since $f_{k}-i<m, n$ is an $m$-repetitive place.

Assume (2) and $i \geq 0$. Then, $n-i$ is an $\left(f_{k+1}-2, f_{k-1}\right)$-shift invariant place by Lemma $8(2)$. Then, $n$ is an $\left(m, f_{k-1}\right)$-shift invariant place since $i+m \leq f_{k+1}-2$. Thus, $n$ is an $m$-repetitive place as $f_{k-1}<m$.

Assume (2) and $i<0$. Then, since $n-i$ is an $\left(f_{k+1}-2, f_{k-1}\right)$-shift invariant place and $m \leq f_{k+1}-2$, it is an $\left(m, f_{k-1}\right)$-shift invariant place. Then $n$ is an $m$-repetitive place, since $f_{k-1}-i<m$. Thus, $n \in \mathcal{R}_{m}$.

COROllary 1. The place 0 is $m$-repetitive for an $m \geq 2$ if $m \notin$ $\bigcup_{k=1}^{\infty}\left\{f_{k}-1, f_{k}\right\}$.

REMARK 3. The "if and only if" statement actually holds in Corollary 1 in place of "if" since we prove later that $H_{0, m} \neq 0$ if $m \in \bigcup_{k=1}^{\infty}\left\{f_{k}-1, f_{k}\right\}$.

Proof (of Corollary 1). Let $i=0$ in (1) of Theorem 1. Then 0 is $m$-repetitive if $f_{k}+1 \leq m \leq f_{k+1}-2$ for some $k \geq 1$. 
Corollary 2. Let $k \geq 2$. The place $n$ is $f_{k}$-repetitive if

$$
W_{k} \prec \widehat{\varepsilon}_{n+1} \widehat{\varepsilon}_{n+2} \ldots \widehat{\varepsilon}_{n+2 f_{k}-3} .
$$

Proof. By (2) of Theorem 1, for any $k \geq 2, n$ is an $f_{k}$-repetitive place if $n-i \equiv_{k+1} f_{k}$ for some $i$ with $i \leq n$ and $-f_{k-2}+1 \leq i \leq f_{k-1}-2$. Since the condition $n-i \equiv_{k+1} f_{k}$ is equivalent to $n-i \equiv_{k+2} f_{k}$ and there is no carry in addition of $-i$ to both sides of $n \equiv_{k+2} f_{k}+i$, the condition $n-i \equiv_{k+1} f_{k}$ is equivalent to $n \equiv_{k+2} f_{k}+i$. Hence, the place $n$ is $f_{k}$-repetitive if $n \equiv_{k+2} j$ for some $j$ with $f_{k-1}+1 \leq j \leq f_{k+1}-2$. By Lemma 6 , this condition is equivalent to $W_{k}$ starting at one of the places in $\left\{n+1, n+2, \ldots, f_{k}-2\right\}$, which completes the proof.

3. Hankel determinants. The aim of this section is to find the value of the Hankel determinants

$$
\begin{aligned}
H_{n, m} & :=H_{n, m}(\varepsilon)=\operatorname{det}\left(\varepsilon_{n+i+j}\right)_{0 \leq i, j \leq m-1}, \\
\bar{H}_{n, m}:=H_{n, m}(\bar{\varepsilon})=\operatorname{det}\left(\bar{\varepsilon}_{n+i+j}\right)_{0 \leq i, j \leq m-1} & (n=0,1, \ldots ; m=1,2, \ldots)
\end{aligned}
$$

for the Fibonacci word $\varepsilon(a, b)$ at $(a, b)=(1,0)$ and $(a, b)=(0,1)$ :

$$
\begin{aligned}
& \varepsilon:=\varepsilon(1,0)=10110101101101 \ldots, \\
& \bar{\varepsilon}:=\varepsilon(0,1)=01001010010010 \ldots
\end{aligned}
$$

It is clear that $H_{n, m}(\varepsilon(a, b))=0$ if $n$ is the $m$-repetitive place in $\varepsilon(a, b)$, where $a, b$ are considered to be two independent variables, and that, in general, $H_{n, m}(\varepsilon(a, b))$ becomes a polynomial in $a$ and $b$ as stated in Remark 1.

In the following lemmas, theorems and corollary, we give parallel statements for $\varepsilon$ and $\bar{\varepsilon}$, while we give the proofs only for $\varepsilon$ since those for $\bar{\varepsilon}$ are similar. The only difference is the starting point, Lemma 5 , where $a-b$ on the right-hand side is 1 for $\varepsilon$ and -1 for $\bar{\varepsilon}$.

We use the following notation: for every subset $S$ of $\{0,1,2,3,4,5\}$, $\chi(k: S)$ is the function on $k \in \mathbb{Z}$ such that

$$
\chi(k: S)= \begin{cases}-1 & \text { if } k \equiv s(\bmod 6) \text { for some } s \in S, \\ 1 & \text { otherwise. }\end{cases}
$$

The following corollary follows from Theorem 1.

Corollary 3. $H_{n, m}=0$ if one of the conditions (1), (2) in Theorem 1 is satisfied. The same statement holds for $\bar{H}_{n, m}$.

Lemma 9. For any $k \geq 2$, we have

$$
\begin{aligned}
& H_{0, f_{k}}=\chi(k: 2,3)\left(H_{0, f_{k-1}}-(-1)^{f_{k-1}} H_{f_{k}-1, f_{k-1}}\right), \\
& \bar{H}_{0, f_{k}}=\chi(k: 1,3,4,5)\left(\bar{H}_{0, f_{k-1}}-(-1)^{f_{k-1}} \bar{H}_{f_{k}-1, f_{k-1}}\right) .
\end{aligned}
$$


Proof. The matrix $\left(\varepsilon_{i+j}\right)_{0 \leq i, j<f_{k}}$ is decomposed into three parts:

$$
\left(\varepsilon_{i+j}\right)_{0 \leq i, j<f_{k}}=\left(\begin{array}{c}
A \\
A^{\prime} \\
B
\end{array}\right),
$$

where

$$
\begin{aligned}
& A=\left(\varepsilon_{i+j}\right)_{0 \leq i<f_{k-2}, 0 \leq j<f_{k}}, \\
& A^{\prime}=\left(\varepsilon_{f_{k-2}}+i+j\right. \\
&)_{0 \leq i<f_{k-3}, 0 \leq j<f_{k}}, \\
& B=\left(\varepsilon_{f_{k-1}+i+j}\right)_{0 \leq i<f_{k-2}, 0 \leq j<f_{k}} .
\end{aligned}
$$

By Lemma 5, the following two subwords of $\varepsilon$ :

$$
\varepsilon_{0} \varepsilon_{1} \ldots \varepsilon_{f_{k-2}+f_{k}-2} \text { and } \varepsilon_{f_{k-1}} \varepsilon_{f_{k-1}+1} \ldots \varepsilon_{f_{k-1}+f_{k-2}+f_{k}-2}
$$

differ only at two places, namely, $\varepsilon_{f_{k}-2} \neq \varepsilon_{f_{k-1}+f_{k}-2}$ and $\varepsilon_{f_{k}-1} \neq$ $\varepsilon_{f_{k-1}+f_{k}-1}$. Thus, we get

$$
B-A=\left(\begin{array}{cccccc} 
& & & & (-1)^{k} & (-1)^{k-1} \\
0 & & & (-1)^{k} & (-1)^{k-1} &
\end{array}\right)
$$

Let $A_{0}, A_{1}, \ldots, A_{f_{k}-1}$ be the columns of the matrix $\left(\begin{array}{c}A \\ A^{\prime}\end{array}\right)$ in order from the left. Since

$$
\begin{aligned}
\left(A_{0} A_{1} \ldots A_{f_{k-2}-2}\right) & =\left(\varepsilon_{i+j}\right)_{0 \leq i<f_{k-1}, 0 \leq j<f_{k-2}-1}, \\
\left(A_{f_{k-1}} A_{f_{k-1}+1} \ldots A_{f_{k}-2}\right) & =\left(\varepsilon_{f_{k-1}+i+j}\right)_{0 \leq i<f_{k-1}, 0 \leq j<f_{k-2}-1}
\end{aligned}
$$

and

$$
\varepsilon_{0} \varepsilon_{1} \ldots \varepsilon_{f_{k-2}+f_{k-1}-3}=\varepsilon_{f_{k-1}} \varepsilon_{f_{k-1}+1} \ldots \varepsilon_{f_{k-1}+f_{k-2}+f_{k-1}-3}
$$

by Lemma 5 , we get

$$
\left(A_{0} A_{1} \ldots A_{f_{k-2}-2}\right)=\left(A_{f_{k-1}} A_{f_{k-1}+1} \ldots A_{f_{k}-2}\right) .
$$

Thus, from (15) and (16) we obtain

$$
\begin{aligned}
& \text { (17) } H_{0, f_{k}}=\operatorname{det}\left(\begin{array}{ccccccc}
A_{0} \ldots & A_{f_{k-1}-1} & A_{f_{k-1}} & \ldots & A_{f_{k}-2} & A_{f_{k}-1} \\
& & & & & (-1)^{k} & (-1)^{k-1} \\
& & & & & (-1)^{k} & (-1)^{k-1}
\end{array}\right. \\
& =\operatorname{det}\left(\begin{array}{ccccccc}
A_{0} & \ldots & A_{f_{k-1}-1} & 0 & \ldots & 0 & A_{f_{k}-1} \\
& & & & (-1)^{k} & (-1)^{k-1} & (-1)^{k-1} \\
& & & \ldots & \ldots & 0 &
\end{array}\right)
\end{aligned}
$$




$$
\begin{aligned}
& =(-1)^{(k-1) f_{k-2}}(-1)^{\left[f_{k-2} / 2\right]} \operatorname{det}\left(A_{0} A_{1} \ldots A_{f_{k-1}-1}\right) \\
& +(-1)^{k f_{k-2}}(-1)^{\left[f_{k-2} / 2\right]+f_{k}-1} \operatorname{det}\left(A_{f_{k}-1} A_{0} A_{1} \ldots A_{f_{k-1}-2}\right) \text {. }
\end{aligned}
$$

Since

$$
\varepsilon_{0} \varepsilon_{1} \ldots \varepsilon_{2 f_{k-1}-3}=\varepsilon_{f_{k}} \varepsilon_{f_{k}+1} \ldots \varepsilon_{f_{k}+2 f_{k-1}-3}
$$

by Lemma 5 , we get

$$
\operatorname{det}\left(A_{f_{k}-1} A_{0} A_{1} \ldots A_{f_{k-1}-2}\right)=\operatorname{det}\left(\varepsilon_{f_{k}-1+i+j}\right)_{0 \leq i, j<f_{k-1}}=H_{f_{k}-1, f_{k-1}} .
$$

Thus we get

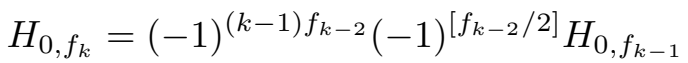

$$
\begin{aligned}
& +(-1)^{k f_{k-2}}(-1)^{\left[f_{k-2} / 2\right]+f_{k}-1} H_{f_{k}-1, f_{k-1}} \\
& =\chi(k: 2,3)\left(H_{0, f_{k-1}}-(-1)^{f_{k-1}} H_{f_{k}-1, f_{k-1}}\right),
\end{aligned}
$$

where we have used the fact that

$$
(-1)^{(k-1) f_{k-2}(-1)^{\left[f_{k-2} / 2\right]}=\chi(k: 2,3)}
$$

Lemma 10. For $k \geq 2$, we have

$$
\begin{aligned}
& H_{f_{k+1}-1, f_{k}}=\chi(k: 1,3,4,5) H_{f_{k+1}-1, f_{k-1}}, \\
& \bar{H}_{f_{k+1}-1, f_{k}}=\chi(k: 2,3) \bar{H}_{f_{k+1}-1, f_{k-1}} .
\end{aligned}
$$

Proof. Just as in the proof of Lemma 9, we decompose the matrix $\left(\varepsilon_{f_{k+1}-1+i+j}\right)_{0 \leq i, j<f_{k}}$ into three parts:

$$
\left(\varepsilon_{f_{k+1}-1+i+j}\right)_{0 \leq i, j<f_{k}}=\left(\begin{array}{c}
A \\
A^{\prime} \\
B
\end{array}\right),
$$

where

$$
\begin{aligned}
A & =\left(\varepsilon_{f_{k+1}-1+i+j}\right)_{0 \leq i<f_{k-2}, 0 \leq j<f_{k}}, \\
A^{\prime} & =\left(\varepsilon_{f_{k+1}-1+f_{k-2}+i+j}\right)_{0 \leq i<f_{k-3}, 0 \leq j<f_{k}}, \\
B & =\left(\varepsilon_{f_{k+1}-1+f_{k-1}+i+j}\right)_{0 \leq i<f_{k-2}, 0 \leq j<f_{k}} .
\end{aligned}
$$

By Lemma 5 , the following two subwords of $\varepsilon$ :

$$
\begin{aligned}
& \varepsilon_{f_{k+1}-1} \varepsilon_{f_{k+1}} \ldots \varepsilon_{f_{k+1}+f_{k-2}+f_{k}-3} \text { and } \\
& \varepsilon_{f_{k+1}-1+f_{k-1}} \varepsilon_{f_{k+1}+f_{k-1}} \ldots \varepsilon_{f_{k+1}+f_{k-1}+f_{k-2}+f_{k}-3}
\end{aligned}
$$

differ only at two places. Namely, $\varepsilon_{f_{k+1}+f_{k}-2} \neq \varepsilon_{f_{k+1}+f_{k-1}+f_{k}-2}$ and $\varepsilon_{f_{k+1}+f_{k}-1} \neq \varepsilon_{f_{k+1}+f_{k-1}+f_{k}-1}$. Therefore, we get

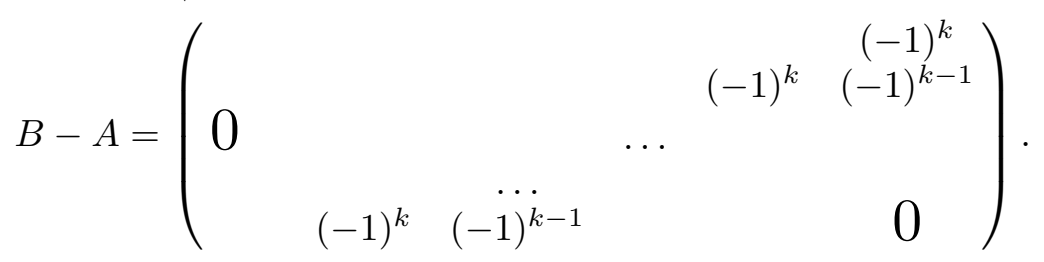


Thus, we have

(18) $\operatorname{det}\left(\varepsilon_{f_{k+1}-1+i+j}\right)_{0 \leq i, j<f_{k}}$

$$
\begin{aligned}
& =\operatorname{det}\left(\begin{array}{cccccccc}
A_{0} & A_{1} & \ldots & A_{f_{k-1}-1} & A_{f_{k-1}} & \ldots & A_{f_{k}-2} & A_{f_{k}-1} \\
& & & & & & & (-1)^{k} \\
& & & & & & (-1)^{k} & (-1)^{k-1} \\
& & & & (-1)^{k} & (-1)^{k-1} & & 0
\end{array}\right) \\
& =(-1)^{k f_{k-2}}(-1)^{\left[f_{k-2} / 2\right]} \operatorname{det}\left(A_{0} A_{1} \ldots A_{f_{k-1}-1}\right) \\
& =\chi(k: 1,3,4,5) H_{f_{k+1}-1, f_{k-1}} \text {. }
\end{aligned}
$$

Lemma 11. For any $k \geq 2$, we have

$$
\begin{aligned}
& H_{f_{k+1}-1, f_{k-1}}=\chi(k: 2,5) H_{0, f_{k-1}}, \\
& \bar{H}_{f_{k+1}-1, f_{k-1}}=\chi(k: 2,5) \bar{H}_{0, f_{k-1}} .
\end{aligned}
$$

Pr o of. Since, by Lemma 5,

$\varepsilon_{f_{k+1}-1} \varepsilon_{f_{k+1}} \ldots \varepsilon_{f_{k+1}+f_{k-1}-2}=\varepsilon_{f_{k+1}+f_{k-1}-1} \varepsilon_{f_{k+1}+f_{k-1}} \ldots \varepsilon_{f_{k+1}+2 f_{k-1}-2}$, we get

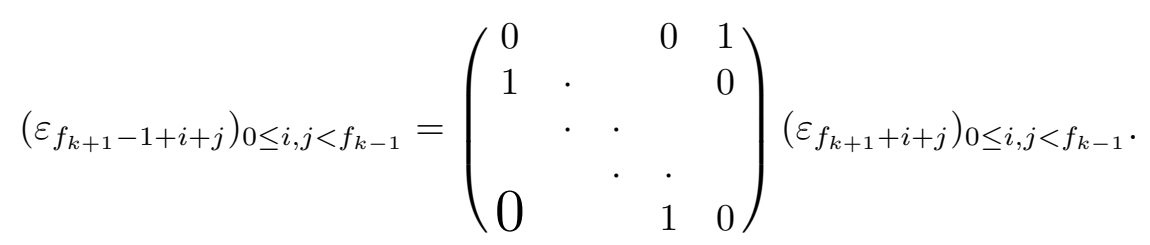

Also, by Lemma 5 ,

$$
\left(\varepsilon_{f_{k+1}+i+j}\right)_{0 \leq i, j<f_{k}}=\left(\varepsilon_{i+j}\right)_{0 \leq i, j<f_{k}} .
$$

Thus we obtain

$$
\begin{aligned}
H_{f_{k+1}-1, f_{k-1}} & =\operatorname{det}\left(\varepsilon_{f_{k+1}-1+i+j}\right)_{0 \leq i, j<f_{k-1}} \\
& =(-1)^{f_{k-1}-1} \operatorname{det}\left(\varepsilon_{f_{k+1}+i+j}\right)_{0 \leq i, j<f_{k-1}} \\
& =\chi(k: 2,5) H_{0, f_{k-1}} .
\end{aligned}
$$

Lemma 12. For any $k \geq 3$, we have

$$
\begin{aligned}
& H_{0, f_{k}}=\chi(k: 2,3) H_{0, f_{k-1}}+\chi(k: 2,4) H_{0, f_{k-2}}, \\
& \bar{H}_{0, f_{k}}=\chi(k: 1,3,4,5) \bar{H}_{0, f_{k-1}}+\chi(k: 0,1,2,3) \bar{H}_{0, f_{k-2}} .
\end{aligned}
$$

P r o of. Clear from Lemmas 9-11.

Lemma 13. For any $k \geq 0$, we have

$$
\begin{aligned}
& H_{0, f_{k}}=\chi(k: 2) f_{k-1}, \\
& \bar{H}_{0, f_{k}}=\chi(k: 1,2,4) f_{k-2} .
\end{aligned}
$$


Proof. We have

$$
\begin{array}{lll}
H_{0, f_{0}}=1, & H_{0, f_{1}}=1, & H_{0, f_{2}}=-2, \\
\bar{H}_{0, f_{0}}=0, & \bar{H}_{0, f_{1}}=-1, & \bar{H}_{0, f_{2}}=-1 .
\end{array}
$$

Thus, the assertion holds for $k=0,1,2$. For $k \geq 3$, we can prove it by induction on $k$ using Lemma 12.

Lemma 14. For any $k \geq 1$, we have

$$
\begin{aligned}
& H_{0, f_{k}-1}=\chi(k: 0,4) f_{k-2}, \\
& \bar{H}_{0, f_{k}-1}=\chi(k: 2,3,4,5) f_{k-3} .
\end{aligned}
$$

Pr o of. Since the matrix $\left(\varepsilon_{i+j}\right)_{0 \leq i, j<f_{k}-1}$ is obtained from $\left(\varepsilon_{i+j}\right)_{0 \leq i, j<f_{k}}$ by removing the last row and the last column, for any $k \geq 2$ we have by (17),

$$
\begin{aligned}
& =\operatorname{det}\left(\begin{array}{cccccccc}
A_{0} & A_{1} & \ldots & A_{f_{k-1}-1} & 0 & \ldots & 0 & 0 \\
& & & & & & & (-1)^{k} \\
& & & & & & (-1)^{k} & (-1)^{k-1} \\
& & & & (-1)^{k} & (-1)^{k-1} & \ldots & 0
\end{array}\right) \\
& =(-1)^{k\left(f_{k-2}-1\right)}(-1)^{\left[\left(f_{k-2}-1\right) / 2\right]} \operatorname{det}\left(A_{0} A_{1} \ldots A_{f_{k-1}-1}\right) \\
& =(-1)^{k\left(f_{k-2}-1\right)}(-1)^{\left[\left(f_{k-2}-1\right) / 2\right]} H_{0, f_{k-1}} .
\end{aligned}
$$

Hence, in view of Lemma 13, we obtain the formula for $H_{0, f_{k}-1}$.

TheOrem 2. For any $m, k \geq 1$ with $f_{k-1}<m \leq f_{k}$ and $n \in \mathbb{N}$ with $n \equiv_{k+1} 0$, we have

$$
\begin{aligned}
& H_{n, m}= \begin{cases}\chi(k: 2) f_{k-1} & \text { if } m=f_{k}, \\
\chi(k: 0,4) f_{k-2} & \text { if } m=f_{k}-1, \\
0 & \text { otherwise, }\end{cases} \\
& \bar{H}_{n, m}= \begin{cases}\chi(k: 1,2,4) f_{k-2} & \text { if } m=f_{k}, \\
\chi(k: 2,3,4,5) f_{k-3} & \text { if } m=f_{k}-1, \\
0 & \text { otherwise. }\end{cases}
\end{aligned}
$$

Proof. By Lemmas 3 and 7, the matrix for $H_{n, m}$ coincides with that for $H_{0, m}$ so that $H_{n, m}=H_{0, m}$. Thus, the first two cases follow from Lemmas 13 and 14. For the last case, by Corollary 1, there exist two identical rows in the matrix $\left(\varepsilon_{i+j}\right)_{0 \leq i, j<m}$, so that $H_{0, m}=0$. 

have

Theorem 3. For any $k, n, i \in \mathbb{N}$ with $n \equiv_{k+1} i$ and $0 \leq i \leq f_{k+1}-1$, we

$$
\begin{aligned}
& H_{n, f_{k}}=\left\{\begin{array}{l}
\chi(k: 2) \chi(k: 1,4)^{i} f_{k-1} \\
\quad \text { if either } \tau_{k+1}(n)=0 \text { and } 0 \leq i<f_{k-1} \\
\text { or } \tau_{k+1}(n)=1 \text { and } 0 \leq i<f_{k}, \\
\chi(k: 1,2,4) f_{k-2} \\
\quad \text { if either } \tau_{k+1}(n)=0 \text { and } i=f_{k-1} \\
\quad \begin{array}{l}
\text { or } i=f_{k+1}-1, \\
\quad \text { otherwise, }
\end{array}
\end{array}\right. \\
& \bar{H}_{n, f_{k}}=\left\{\begin{array}{l}
\chi(k: 1,2,4) \chi(k: 1,4)^{i} f_{k-2} \\
\quad \text { if either } \tau_{k+1}(n)=0 \text { and } 0 \leq i<f_{k-1} \\
\quad \text { or } \tau_{k+1}(n)=1 \text { and } 0 \leq i<f_{k}, \\
\chi(k: 2) f_{k-3} \\
\quad \text { if either } \tau_{k+1}(n)=0 \text { and } i=f_{k-1} \\
\text { or } i=f_{k+1}-1, \\
\quad \text { otherwise. }
\end{array}\right.
\end{aligned}
$$

Proof. The assertion holds for $k=0$. Let $k \geq 1$.

Assume that either $\tau_{k+1}(n)=0$ and $0 \leq i<f_{k-1}$ or $\tau_{k+1}(n)=1$ and $0 \leq i<f_{k}$. Then by Lemmas 3 and 7 we have

$$
\begin{aligned}
\varepsilon_{i+j} & =\varepsilon_{n+j} & & \left(j=0,1, \ldots, f_{k}-i-1\right), \\
\varepsilon_{i+j-f_{k}} & =\varepsilon_{n+j} & & \left(j=f_{k}-i, f_{k}, \ldots, 2 f_{k}-2\right), \\
\varepsilon_{j} & =\varepsilon_{j+f_{k}} & & \left(j=0,1, \ldots, f_{k}-1\right) .
\end{aligned}
$$

Hence, the columns of the matrix $\left(\varepsilon_{n+h+j}\right)_{0 \leq h, j<f_{k}}$ coincide with those of $\left(\varepsilon_{h+j}\right)_{0 \leq h, j \leq f_{k}}$. The $j$ th column of the former is the $(i+j)\left(\bmod f_{k}\right)$ th column of the latter for $j=0, \ldots, f_{k}-1$. Therefore, we get $H_{n, f_{k}}=$ $(-1)^{i\left(f_{k}-i\right)} H_{0, f_{k}}$, which leads to the first case of our theorem by Theorem 2 .

Assume that $i=f_{k+1}-1$. Then $H_{n, f_{k}}=H_{f_{k+1}-1, f_{k}}$ by Lemmas 3 and 7 . Thus, by Lemmas 10-12 we get

$$
H_{n, f_{k}}=\chi(k: 1,2,4) f_{k-2} .
$$

Assume that $\tau_{k+1}(n)=0$ and $i=f_{k-1}$. Then, since $n \equiv_{k+2} i$, we have $H_{n, f_{k}}=H_{f_{k-1}, f_{k}}$ by Lemmas 3 and 7 . By Lemma 1 ,

$$
\begin{aligned}
& \xi:=\varepsilon_{f_{k-1}} \varepsilon_{f_{k-1}+1} \ldots \varepsilon_{f_{k-1}+2 f_{k}-2} \prec_{1} W_{k-2} W_{k-1} W_{k} W_{k-1} W_{k-2}, \\
& \eta:=\varepsilon_{f_{k+1}-1} \varepsilon_{f_{k+1}} \ldots \varepsilon_{f_{k+1}+2 f_{k}-3} \prec_{f_{k}} W_{k-2} W_{k-1} W_{k} W_{k-1} W_{k-2} .
\end{aligned}
$$

Since the last letter of $\eta$ comes one letter before the last letter of the palindrome word $W_{k-2} W_{k-1} W_{k} W_{k-1} W_{k-2}$, it follows that $\xi$ is the mirror image of $\eta$, so that 


$$
\begin{aligned}
& \left(\varepsilon_{f_{k-1}+i+j}\right)_{0 \leq i, j<f_{k}}
\end{aligned}
$$

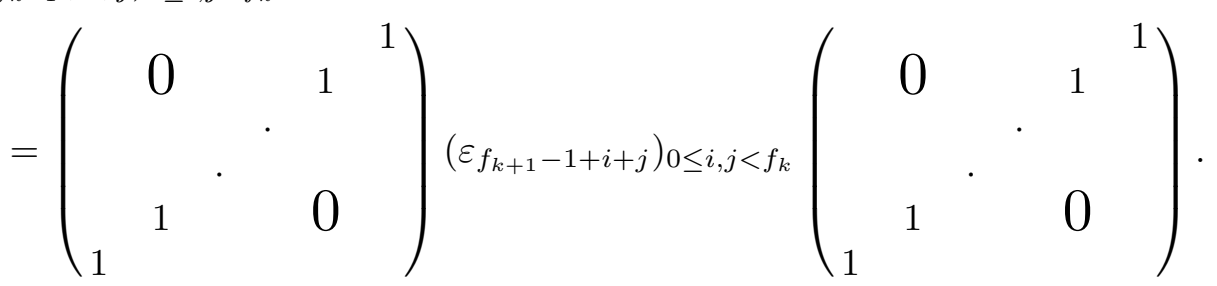

Thus, we obtain $H_{f_{k-1}, f_{k}}=H_{f_{k+1}-1, f_{k}}$ and

$$
H_{n, f_{k}}=\chi(k: 1,2,4) f_{k-2} .
$$

Assume that $n$ does not belong to the above two cases. Then, since $\tau_{k+1}(n)=1$ implies $i<f_{k}$, we have the following condition:

$$
\tau_{k+1}(n)=0 \quad \text { and } \quad f_{k-1}+1 \leq i \leq f_{k+1}-2 .
$$

This condition is nonempty only if $k \geq 2$, which we assume. Then the condition (2) of Theorem 1 is satisfied with $f_{k}$ (resp. $i-f_{k}$ ) in place of $m$ (resp. $i$ ). Thus, by Corollary $3, H_{n, f_{k}}=0$.

Lemma 15. For any $k, n, i \in \mathbb{N}$ with $k \geq 1$ and $n \equiv_{k+1} i$, assume that either $\tau_{k+1}(n)=0$ and $0 \leq i<f_{k-1}$ or $\tau_{k+1}(n)=1$ and $0 \leq i<f_{k}$. Then

$$
\begin{aligned}
& H_{n, f_{k}-1}= \begin{cases}\chi(k: 0,4) f_{k-2} & (i=0), \\
\chi(k: 2,3) \chi(k: 1,2,4,5)^{i} H_{i+f_{k}, f_{k-1}-1} & \\
\quad+\chi(k: 1,2,3,5) \chi(k: 1,4)^{i} f_{k-2} & \left(0<i \leq f_{k-2}\right), \\
\chi(k: 2,3) \chi(k: 1,2,4,5)^{i} H_{i+f_{k}, f_{k-1}-1} & \left(f_{k-2}<i \leq f_{k-1}\right), \\
\chi(k: 0,4) \chi(k: 1,4)^{i} f_{k-2} & \left(f_{k-1}<i<f_{k}\right),\end{cases} \\
& \bar{H}_{n, f_{k}-1}= \begin{cases}\chi(k: 2,3,4,5) f_{k-3} & (i=0), \\
\chi(k: 1,3,4,5) \chi(k: 1,2,4,5)^{i} \bar{H}_{i+f_{k}, f_{k-1}-1} & \\
\quad+\chi(k: 0,1) \chi(k: 1,4)^{i} f_{k-3} & \left(0<i \leq f_{k-2}\right), \\
\chi(k: 1,3,4,5) \chi(k: 1,2,4,5)^{i} \bar{H}_{i+f_{k}, f_{k-1}-1} & \left(f_{k-2}<i \leq f_{k-1}\right), \\
\chi(k: 2,3,4,5) \chi(k: 1,4)^{i} f_{k-3} & \left(f_{k-1}<i<f_{k}\right) .\end{cases}
\end{aligned}
$$

Proof. If $i=0$, then the statement follows from Theorem 2. Let

$$
\begin{aligned}
& A_{j}={ }^{t}\left(\varepsilon_{j}, \varepsilon_{j+1}, \ldots, \varepsilon_{j+f_{k-1}-1}\right), \\
& A_{j}^{\prime}={ }^{t}\left(\varepsilon_{j}, \varepsilon_{j+1}, \ldots, \varepsilon_{j+f_{k-1}-2}\right), \\
& B_{j}^{\prime}={ }^{t}\left(\varepsilon_{j+f_{k-1}}, \varepsilon_{j+f_{k-1}+1}, \ldots, \varepsilon_{j+f_{k}-1}\right) \quad(j=0,1, \ldots) .
\end{aligned}
$$

Then, by the same argument as in the proof of Theorem 3 , we obtain

$$
\begin{aligned}
H_{n, f_{k}-1} & =\operatorname{det}\left(\begin{array}{c}
A_{i} \ldots A_{f_{k}-1} A_{0} \ldots A_{i-2} \\
B_{i}^{\prime} \ldots B_{f_{k}-1}^{\prime} B_{0}^{\prime} \ldots B_{i-2}^{\prime}
\end{array}\right) \\
& =(-1)^{(i-1)\left(f_{k}-i\right)} \operatorname{det}\left(\begin{array}{c}
A_{0} \ldots A_{i-2} A_{i} \ldots A_{f_{k}-1} \\
B_{0}^{\prime} \ldots B_{i-2}^{\prime} B_{i}^{\prime} \ldots B_{f_{k}-1}^{\prime}
\end{array}\right) .
\end{aligned}
$$


Therefore, if $f_{k-2}<i \leq f_{k-1}$, then by the same argument as for (17), we obtain

$$
=\operatorname{det}\left(\begin{array}{ccccc}
A_{0} \ldots A_{i-2} A_{i} \ldots A_{f_{k-1}-1} & 0 & \ldots & 0 & A_{f_{k}-1} \\
& & & (-1)^{k} & (-1)^{k-1} \\
0 & & \ldots & (-1)^{k-1} & 0
\end{array}\right) .
$$

Since by Lemma 5 ,

we get

$$
A_{f_{k}-1}-A_{f_{k-2}-1}=\left(\begin{array}{c}
0 \\
\vdots \\
0 \\
(-1)^{k}
\end{array}\right)
$$

$$
\begin{aligned}
& (-1)^{(i-1)\left(f_{k}-i\right)} H_{n, f_{k}-1} \\
& =\operatorname{det}\left(\begin{array}{ccccc}
A_{0}^{\prime} \ldots A_{i-2}^{\prime} A_{i}^{\prime} \ldots A_{f_{k-1}-1}^{\prime} & 0 & \ldots & 0 & 0 \\
* \ldots * * \ldots * & 0 & \ldots & 0 & (-1)^{k} \\
& & & (-1)^{k} & (-1)^{k-1} \\
0 & & \ldots & & 0
\end{array}\right) \\
& =(-1)^{k f_{k-2}}(-1)^{\left[f_{k-2} / 2\right]} \operatorname{det}\left(A_{0}^{\prime} \ldots A_{i-2}^{\prime} A_{i}^{\prime} \ldots A_{f_{k-1}-1}^{\prime}\right) \\
& =\chi(k: 1,3,4,5)(-1)^{(i-1)\left(f_{k-1}-i\right)} H_{i+f_{k}, f_{k-1}-1} \text {. }
\end{aligned}
$$

Thus we obtain

$$
H_{n, f_{k}-1}=\chi(k: 2,3) \chi(k: 1,2,4,5)^{i} H_{i+f_{k}, f_{k-1}-1} .
$$

Assume that $f_{k-1}<i<f_{k}$. Then as above we have

$$
\begin{aligned}
& (-1)^{(i-1)\left(f_{k}-i\right)} H_{n, f_{k}-1}
\end{aligned}
$$

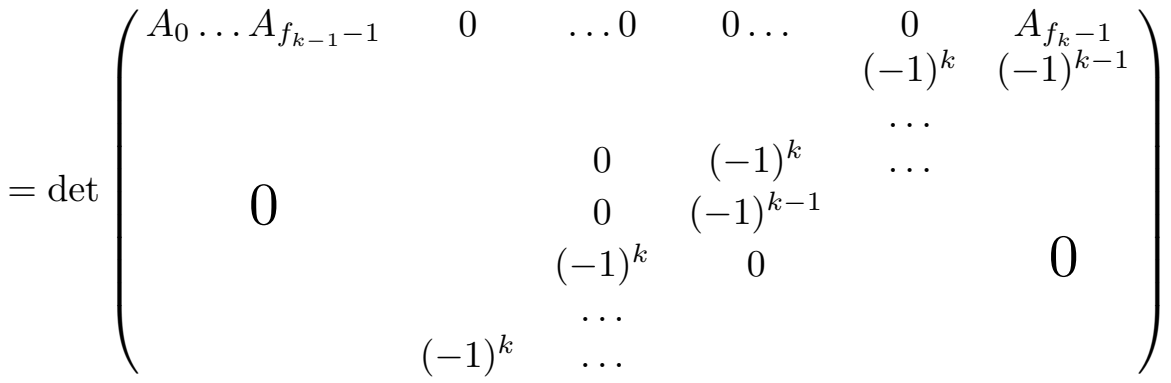

$$
\begin{aligned}
& =(-1)^{k\left(i-f_{k-1}-1\right)+(k-1)\left(f_{k}-i\right)+\left[\left(f_{k-2}-1\right) / 2\right]} \operatorname{det}\left(A_{0} \ldots A_{f_{k-1}-1}\right) \text {. }
\end{aligned}
$$


Hence, by Lemma 13,

$$
H_{n, f_{k}-1}=\chi(k: 0,3,4) \chi(k: 1,4)^{i} H_{0, f_{k-1}}=\chi(k: 0,4) \chi(k: 1,4)^{i} f_{k-2} .
$$

Assume that $0<i<f_{k-2}$. Then, since $A_{i-1+f_{k-1}}=A_{i-1}$, by the same arguments as above we get

$$
\begin{aligned}
& (-1)^{(i-1)\left(f_{k}-i\right)} H_{n, f_{k}-1}
\end{aligned}
$$

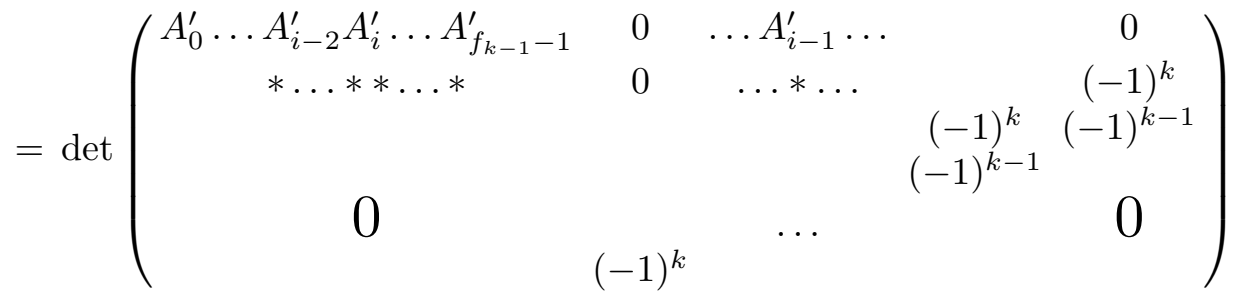

$$
\begin{aligned}
& =(-1)^{k f_{k-2}}(-1)^{\left[f_{k-2} / 2\right]} \operatorname{det}\left(A_{0}^{\prime} \ldots A_{i-2}^{\prime} A_{i}^{\prime} \ldots A_{f_{k-1}-1}^{\prime}\right) \\
& +(-1)^{k(i-1)+(k-1)\left(f_{k-2}-i\right)}(-1)^{i-1+\left[\left(f_{k-2}-1\right) / 2\right]} \\
& \times \operatorname{det}\left(A_{0} \ldots A_{i-2} A_{i} \ldots A_{f_{k-1}-1} A_{i-1}\right) \text {. }
\end{aligned}
$$

Since

$$
\operatorname{det}\left(A_{0} \ldots A_{i-2} A_{i} \ldots A_{f_{k-1}-1} A_{i-1}\right)=(-1)^{f_{k-1}-i} H_{0, f_{k-1}},
$$

by Lemma 13 we obtain

$$
\begin{aligned}
H_{n, f_{k}-1}= & \chi(k: 2,3) \chi(k: 1,2,4,5)^{i} H_{i+f_{k}, f_{k-1}-1} \\
& +\chi(k: 1,2,3,5) \chi(k: 1,4)^{i} f_{k-2} .
\end{aligned}
$$

Note that (21) holds also for $i=f_{k-2}$ since in this case,

$$
\begin{aligned}
H_{n, f_{k}-1}= & (-1)^{k\left(f_{k-2}-1\right)}(-1)^{f_{k-2}-1+\left[\left(f_{k-2}-1\right) / 2\right]} \\
& \times \operatorname{det}\left(A_{0} \ldots A_{f_{k-2}-2} A_{f_{k-2}} \ldots A_{f_{k-1}-2} A_{f_{k}-1}\right)
\end{aligned}
$$

and

$$
A_{f_{k}-1}=A_{f_{k-1}-1}+{ }^{t}\left(0, \ldots, 0,(-1)^{k}\right) .
$$

Lemma 16. For any $k, n, i \in \mathbb{N}$ with $k \geq 1$ and $n \equiv_{k+1} i$, assume that either $\tau_{k+1}(n)=0$ and $0 \leq i<f_{k-1}$ or $\tau_{k+1}(n)=1$ and $0 \leq i<f_{k}$. Then

$$
\begin{aligned}
& H_{n, f_{k}-1}= \begin{cases}\chi(k: 0,4) f_{k-2} & (i=0), \\
\chi(k: 1,2,3,5) \chi(k: 1,4)^{i} f_{k-3} & \left(0<i \leq f_{k-1}\right), \\
\chi(k: 0,4) \chi(k: 1,4)^{i} f_{k-2} & \left(f_{k-1}<i<f_{k}\right),\end{cases} \\
& \bar{H}_{n, f_{k}-1}= \begin{cases}\chi(k: 2,3,4,5) f_{k-3} & (i=0), \\
\chi(k: 0,1) \chi(k: 1,4)^{i} f_{k-4} & \left(0<i \leq f_{k-1}\right), \\
\chi(k: 2,3,4,5) \chi(k: 1,4)^{i} f_{k-3} & \left(f_{k-1}<i<f_{k}\right) .\end{cases}
\end{aligned}
$$


Pr o of. The first and third cases have already been proved in Lemma 15. Consider the second case where $0<i \leq f_{k-1}$. We divide it into two subcases, and use induction on $k$.

CASE $1: i=1$. If $k=1$, then

$$
H_{n, f_{k-1}}=H_{n, 1}=\varepsilon_{n}=0
$$

since $n \equiv_{2} 1$ and $\tau_{0}(n)=1$. On the other hand, $f_{k-3}=f_{-2}=0$, and hence, we get the statement. Assume that $k \geq 2$ and the assertion holds for $k-1$. Then, by Lemma 15 and the induction hypothesis, we get

$$
\begin{aligned}
H_{n, f_{k}-1}= & \chi(k: 2,3) \chi(k: 1,2,4,5)^{i} H_{i+f_{k}, f_{k-1}-1} \\
& +\chi(k: 1,2,3,5) \chi(k: 1,4)^{i} f_{k-2} \\
= & \chi(k: 1,3,4,5) H_{1+f_{k}, f_{k-1}-1}+\chi(k: 2,3,4,5) f_{k-2} \\
= & \chi(k: 1,3,4,5) \chi(k-1: 2,3,4,5) f_{k-4}+\chi(k: 2,3,4,5) f_{k-2} \\
= & \chi(k: 0,1) f_{k-4}+\chi(k: 2,3,4,5) f_{k-2} \\
= & \chi(k: 2,3,4,5) f_{k-3},
\end{aligned}
$$

which is the desired statement.

CASE 2: $i \geq 2$. If $f_{k-2}<i \leq f_{k-1}$, then it follows from the third case and then the fourth case of Lemma 15 that

$$
\begin{aligned}
H_{n, f_{k}-1} & =\chi(k: 2,3) \chi(k: 1,2,4,5)^{i} H_{i+f_{k}, f_{k-1}-1} \\
& =\chi(k: 2,3) \chi(k: 1,2,4,5)^{i} \chi(k-1: 0,4) \chi(k-1: 1,4)^{i} f_{k-3} \\
& =\chi(k: 1,2,3,5) \chi(k: 1,4)^{i} f_{k-3} .
\end{aligned}
$$

Assume that $i \leq f_{k-2}$ and the statement holds for $k-1$. Then by Lemma 15 , we get

$$
\begin{aligned}
H_{n, f_{k}-1}= & \chi(k: 2,3) \chi(k: 1,2,4,5)^{i} H_{i+f_{k}, f_{k-1}-1} \\
& +\chi(k: 1,2,3,5) \chi(k: 1,4)^{i} f_{k-2} \\
= & \chi(k: 2,3) \chi(k: 1,2,4,5)^{i} \chi(k-1: 1,2,3,5) \chi(k-1: 1,4)^{i} f_{k-4} \\
& +\chi(k: 1,2,3,5) \chi(k: 1,4)^{i} f_{k-2} \\
= & \chi(k: 0,4) \chi(k: 1,4)^{i} f_{k-4}+\chi(k: 1,2,3,5) \chi(k: 1,4)^{i} f_{k-2} \\
= & \chi(k: 1,2,3,5) \chi(k: 1,4)^{i} f_{k-3} .
\end{aligned}
$$

Lemma 17. For any $k, n \in \mathbb{N}$ with $k \geq 2$ and $\tau_{k+1}(n)=0$, we have

$$
\begin{aligned}
H_{n, f_{k}-1} & = \begin{cases}\chi(k: 2,3,4,5) f_{k-3} & \left(n \equiv_{k+1} f_{k-1}\right), \\
\chi(k: 0,4) f_{k-2} & \left(n \equiv_{k+1} f_{k-1}+1\right),\end{cases} \\
\bar{H}_{n, f_{k}-1} & = \begin{cases}\chi(k: 0,4) f_{k-4} & \left(n \equiv_{k+1} f_{k-1}\right), \\
\chi(k: 2,3,4,5) f_{k-3} & \left(n \equiv_{k+1} f_{k-1}+1\right) .\end{cases}
\end{aligned}
$$


Proof. Assume that $n \equiv_{k+1} f_{k-1}$. Then since $\tau_{k+1}(n)=0$, we have $n \equiv_{k+2} f_{k-1}$. Therefore, by Lemmas 3 and 7 , we get

$$
H_{n, f_{k}-1}=\operatorname{det}\left(\begin{array}{c}
A_{f_{k-1}} \ldots A_{f_{k}-1} A_{f_{k}} \ldots A_{f_{k+1}-2} \\
B_{f_{k-1}}^{\prime} \ldots B_{f_{k}-1}^{\prime} B_{f_{k}}^{\prime} \ldots B_{f_{k+1}-2}^{\prime}
\end{array}\right)
$$

where we use the notation (20). By Lemma 5, the following two subwords of $\varepsilon$ :

$$
\varepsilon_{n} \varepsilon_{n+1} \ldots \varepsilon_{n+f_{k-2}+f_{k}-3} \text { and } \varepsilon_{n+f_{k-1}} \varepsilon_{n+f_{k-1}+1} \ldots \varepsilon_{n+f_{k-1}+f_{k-2}+f_{k}-3}
$$

differ only at two places, namely, at the $\left(f_{k}-2-f_{k-1}\right)$ th and the $\left(f_{k}-1-\right.$ $\left.f_{k-1}\right)$ th places. Hence, we have

$$
\begin{aligned}
& H_{n, f_{k}-1}=\operatorname{det}\left(\begin{array}{c}
A_{f_{k-1}} \ldots A_{f_{k}-1} A_{f_{k}} \ldots A_{f_{k+1}-2} \\
B_{f_{k-1}}^{\prime} \ldots B_{f_{k}-1}^{\prime} B_{f_{k}}^{\prime} \ldots B_{f_{k+1}-2}^{\prime}
\end{array}\right) \\
& =\operatorname{det}\left(\begin{array}{ccccc}
A_{f_{k-1}} & \ldots & \ldots & A_{f_{k}-1} & A_{f_{k}} \ldots A_{f_{k+1}-2} \\
& & (-1)^{k} & (-1)^{k-1} & \\
0 & & (-1)^{k-1} & & \\
(-1)^{k} & \ldots & \ldots & & 0
\end{array}\right) \text {. }
\end{aligned}
$$

By adding the first $f_{k-2}-1$ columns and subtracting the last $f_{k-2}-1$ columns to and from the column beginning by $A_{f_{k}-1}$, we get the column

$$
{ }^{t}\left(A_{f_{k}-1} 0 \ldots 0\right)+{ }^{t}\left((-1)^{k-1} 0 \ldots 0(-1)^{k} 0 \ldots 0\right),
$$

where $(-1)^{k}$ is at the $\left(f_{k-2}-1\right)$ th place. Since, by Lemma 5 ,

$$
\begin{aligned}
\left(A_{f_{k-1}} \ldots A_{f_{k}-2}\right)- & \left(A_{2 f_{k-1}} \ldots A_{f_{k}-2}\right) \\
& =\left(\begin{array}{rrrrc} 
& & & (-1)^{k-1} \\
& 0 & & (-1)^{k-1} & (-1)^{k} \\
& & \ldots & \ldots & \\
(-1)^{k-1} & (-1)^{k} & \ldots & & 0 \\
(-1)^{k} & & & &
\end{array}\right),
\end{aligned}
$$

we get

$$
\begin{aligned}
& H_{n, f_{k}-1} \\
& =(-1)^{k\left(f_{k-2}-1\right)}(-1)^{f_{k-1}\left(f_{k-2}-1\right)+\left[\left(f_{k-2}-1\right) / 2\right]} \\
& \quad \times\left\{\operatorname{det}\left(A_{f_{k}-1} A_{f_{k}} \ldots A_{f_{k+1}-2}\right)+(-1)^{k-1} \operatorname{det}\left(A_{f_{k}}^{\prime \prime} \ldots A_{f_{k+1}-2}^{\prime \prime}\right)\right. \\
& \left.\quad+(-1)^{k+f_{k-2}-1} \operatorname{det}\left(A_{f_{k}}^{\prime \prime \prime} \ldots A_{f_{k+1}-2}^{\prime \prime \prime}\right)\right\}
\end{aligned}
$$


where

$$
\begin{aligned}
A_{j}^{\prime \prime} & :={ }^{t}\left(\varepsilon_{j+1} \ldots \varepsilon_{j+f_{k-1}-1}\right), \\
A_{j}^{\prime \prime \prime} & ={ }^{t}\left(\varepsilon_{j} \ldots \varepsilon_{j+f_{k-2}-2} \varepsilon_{j+f_{k-2}} \ldots \varepsilon_{j+f_{k-1}-1}\right) .
\end{aligned}
$$

Here, we have

$$
\begin{aligned}
\operatorname{det}\left(A_{f_{k}-1} A_{f_{k}} \ldots A_{f_{k+1}-2}\right) & =H_{f_{k}-1, f_{k-1}}, \\
\operatorname{det}\left(A_{f_{k}}^{\prime \prime} \ldots A_{f_{k+1}-2}^{\prime \prime}\right) & =H_{f_{k}+1, f_{k-1}-1},
\end{aligned}
$$

and by Lemma 5 ,

$$
\begin{aligned}
& \operatorname{det}\left(A_{f_{k}}^{\prime \prime \prime} \ldots A_{f_{k+1}-2}^{\prime \prime \prime}\right) \\
& =\left(\begin{array}{ccccc} 
& 0 & & \\
& & & & (-1)^{k-1} \\
& & & (-1)^{k-1} & (-1)^{k} \\
A_{f_{k}}^{\prime \prime \prime} \ldots A_{f_{k}+f_{k-2}-1}^{\prime \prime \prime} & & \cdots & \ldots & \\
& (-1)^{k-1} & \cdots & \cdots & \\
& (-1)^{k} & & & 0
\end{array}\right) \\
& =\left(\begin{array}{ccccc}
C_{f_{k}} & & & & \\
\vdots & & 0 & & \\
C_{f_{k}+f_{k-2}-2} & & & & (-1)^{k-1} \\
C_{f_{k}+f_{k-2}} & & & (-1)^{k-1} & (-1)^{k} \\
& & & & \\
\vdots & & \cdots & \cdots & \\
& & \cdots & \cdots & \\
C_{f_{k+1}-1} & (-1)^{k-1} & \cdots & & 0
\end{array}\right),
\end{aligned}
$$

where we put

$$
C_{j}=\left(\varepsilon_{j} \varepsilon_{j+1} \ldots \varepsilon_{j+f_{k-2}-1}\right) .
$$

Since $C_{f_{k}+f_{k-2}+j}=C_{f_{k}+j}\left(j=0,1, \ldots, f_{k-3}-2\right)$ by Lemma 5 , we have $\operatorname{det}\left(A_{f_{k}}^{\prime \prime \prime} \ldots A_{f_{k+1}-2}^{\prime \prime \prime}\right)$

$$
=(-1)^{(k-1)\left(f_{k-3}-1\right)+f_{k-3}-1+\left[\left(f_{k-3}-1\right) / 2\right]} \operatorname{det}\left(\begin{array}{c}
C_{f_{k}} \\
\vdots \\
C_{f_{k}+f_{k-2}-2} \\
C_{f_{k+1}-1}
\end{array}\right) .
$$


Moreover it follows from Lemma 5 that

$$
\operatorname{det}\left(\begin{array}{c}
C_{f_{k}} \\
\vdots \\
C_{f_{k}+f_{k-2}-2} \\
C_{f_{k+1}-1}
\end{array}\right)=\operatorname{det}\left(\begin{array}{c}
C_{f_{k+1}} \\
\vdots \\
C_{f_{k+1}+f_{k-2}-2} \\
C_{f_{k+1}-1}
\end{array}\right)=(-1)^{f_{k-2}-1} H_{f_{k+1}-1, f_{k-2}},
$$

which implies

$$
\operatorname{det}\left(A_{f_{k}}^{\prime \prime \prime} \ldots A_{f_{k+1}-2}^{\prime \prime \prime}\right)=\chi(k: 0,3,5) H_{f_{k+1}-1, f_{k-2}} .
$$

Thus by (22), (23), Theorem 3 and Lemma 16, we obtain

$$
\begin{aligned}
H_{n, f_{k}-1}= & \chi(k: 4) H_{f_{k}-1, f_{k-1}}+\chi(k: 0,2) H_{f_{k}+1, f_{k-1}-1} \\
& +\chi(k: 1,3,4) H_{f_{k+1}-1, f_{k-2}} \\
= & \chi(k: 2,3,4,5) f_{k-3}+\chi(k: 2,3,4,5) f_{k-4}+\chi(k: 0,1) f_{k-4} \\
= & \chi(k: 2,3,4,5) f_{k-3},
\end{aligned}
$$

which is the first case of our lemma.

To prove the second case, assume that $n \equiv_{k+1} f_{k-1}+1$. Then as above we get

$$
\begin{aligned}
& H_{n, f_{k}-1}=\operatorname{det}\left(\begin{array}{c}
A_{f_{k-1}+1} \ldots A_{f_{k}-1} A_{f_{k}} \ldots A_{f_{k+1}-1} \\
B_{f_{k-1}+1}^{\prime} \ldots B_{f_{k}-1}^{\prime} B_{f_{k}}^{\prime} \ldots B_{f_{k+1}-1}^{\prime}
\end{array}\right) \\
& =\operatorname{det}\left(\begin{array}{ccccc}
A_{f_{k-1}+1} & \ldots & \ldots & A_{f_{k}-1} & A_{f_{k}} \ldots A_{f_{k+1}-1} \\
& & \begin{array}{c}
(-1)^{k} \\
(-1)^{k-1}
\end{array} & & \\
0 & & \ldots & & 0 \\
(-1)^{k-1} & \ldots & & & \\
(-1)^{k-1} & & & &
\end{array}\right) \\
& =(-1)^{(k-1)\left(f_{k-2}-1\right)}(-1)^{\left(f_{k-2}-1\right) f_{k-1}+\left[\left(f_{k-2}-1\right) / 2\right]} \\
& \times \operatorname{det}\left(A_{f_{k}} \ldots A_{f_{k+1}-1}\right) \text {. }
\end{aligned}
$$

Therefore, by Theorem 3 we get

$$
H_{n, f_{k}-1}=\chi(k: 0,3,4) \chi(k-1: 2) f_{k-2}=\chi(k: 0,4) f_{k-2} .
$$

TheOrem 4. For any $k, n, i \in \mathbb{N}$ with $k \geq 1, n \equiv_{k+1} i$ and $0 \leq i<f_{k+1}$, we have 


$$
\begin{aligned}
& H_{n, f_{k}-1}= \begin{cases}\chi(k: 0,4) f_{k-2} & (i=0), \\
\chi(k: 1,2,3,5) \chi(k: 1,4)^{i} f_{k-3} & \left(0<i \leq f_{k-1}\right), \\
\chi(k: 0,4) \chi(k: 1,4)^{i} f_{k-2} & \left(f_{k-1}<i<f_{k}\right. \\
\chi(k: 0,4) f_{k-2} & \text { and } \left.\tau_{k+1}(n)=1\right), \\
& \left(i=f_{k-1}+1\right. \\
\text { and } \left.\tau_{k+1}(n)=0\right), & (\text { otherwise }),\end{cases} \\
& \bar{H}_{n, f_{k}-1}= \begin{cases}\chi(k: 2,3,4,5) f_{k-3} & (i=0), \\
\chi(k: 0,1) \chi(k: 1,4)^{i} f_{k-4} & \left(0<i \leq f_{k-1}\right), \\
\chi(k: 2,3,4,5) \chi(k: 1,4)^{i} f_{k-3} & \left(f_{k-1}<i<f_{k}\right. \\
\chi(k: 2,3,4,5) f_{k-3} & \text { and } \left.\tau_{k+1}(n)=1\right), \\
& \left(i=f_{k-1}+1\right. \\
0 & \text { and } \left.\tau_{k+1}(n)=0\right),\end{cases}
\end{aligned}
$$

Proof. The first four cases follow from Lemmas 16 and 17 . Note that for $i=f_{k-1}$, the assertions in these lemmas coincide, so that $H_{n, f_{k}-1}$ is independent of $\tau_{k+1}(n)$. Consider the last case, where $\tau_{k+1}(n)=0$ and $f_{k-1}+2 \leq i \leq f_{k+1}-1$. We may assume that $k \geq 2$. Then, with $m=f_{k}-1$ and $i-f_{k}$ in place of $i$ there, the condition (2) of Theorem 1 is satisfied. Therefore by Theorem $1, n \in \mathcal{R}_{m}$, which implies that $H_{n, f_{k}-1}=0$.

Lemma 18. For any $n, m \in \mathbb{N}$ such that $f_{k-2}+1 \leq m \leq f_{k}-2, i \leq n$ and $n-i \equiv_{k+1} 0$ for some $i, k \in \mathbb{Z}$ with $k \geq 2$ and $m+i=f_{k}$, we have

$$
\begin{aligned}
& H_{n, m}=\chi(k: 2) \chi(k: 3,4,5)^{i}(-1)^{[i / 2]} f_{k-3}, \\
& \bar{H}_{n, m}=\chi(k: 1,4) \chi(k: 0,1,2)^{i}(-1)^{[i / 2]} f_{k-3} .
\end{aligned}
$$

Proof. First, we consider the case $i<f_{k-2}$. By arguments similar to those used in the proof of Lemma 15, we get, with the notation (20),

$$
H_{n, m}=\operatorname{det}\left(\begin{array}{ccccccc}
A_{i} A_{i+1} & \ldots & A_{f_{k-1}+i-1} & 0 & \ldots & 0 & A_{f_{k}-1} \\
& & & & & (-1)^{k} & (-1)^{k-1} \\
& 0 & & & & \cdots & 0 \\
& & (-1)^{k} & (-1)^{k-1} & & &
\end{array}\right) .
$$

Therefore, by Theorems 3 and 4 ,

$$
\begin{aligned}
H_{n, m}= & (-1)^{k\left(f_{k-2}-i+1\right)+\left[\left(f_{k-2}-i+1\right) / 2\right]} H_{i, f_{k-1}-1} \\
& +(-1)^{(k-1)\left(f_{k-2}-i\right)+\left[\left(f_{k-2}-i\right) / 2\right]} H_{i, f_{k-1}} \\
= & \chi(k: 2) \chi(k: 3,4,5)^{i}(-1)^{[i / 2]}\left(-f_{k-4}+f_{k-2}\right) \\
= & \chi(k: 2) \chi(k: 3,4,5)^{i}(-1)^{[i / 2]} f_{k-3} .
\end{aligned}
$$

If $i=f_{k-2}$, then the statement follows from Theorem 3 . 
Finally, we consider the case $f_{k-2}<i<f_{k-1}$. Then, setting

$$
A_{j}^{r}={ }^{t}\left(\varepsilon_{j} \varepsilon_{j+1} \ldots \varepsilon_{j+r-1}\right),
$$

by Theorem 3 we obtain

$$
\begin{aligned}
& H_{n, m}=\operatorname{det}\left(A_{i}^{f_{k}-i} A_{i+1}^{f_{k}-i} \ldots A_{f_{k}-1}^{f_{k}-i}\right)
\end{aligned}
$$

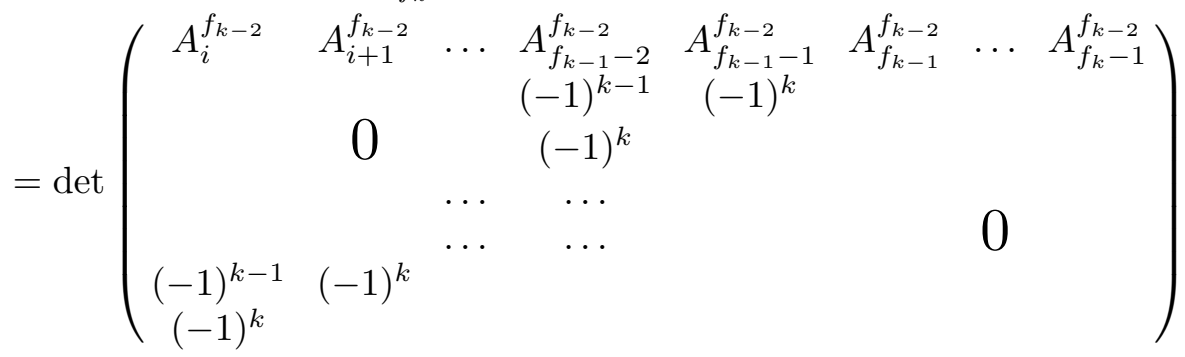

$$
\begin{aligned}
& =(-1)^{k\left(f_{k-1}-i\right)}(-1)^{\left(f_{k-1}-i\right) f_{k-2}+\left[\left(f_{k-1}-i\right) / 2\right]} H_{f_{k-1}, f_{k-2}} \\
& =\chi(k: 2) \chi(k: 3,4,5)^{i}(-1)^{[i / 2]} f_{k-3} .
\end{aligned}
$$

Lemma 19. For any $n, m \in \mathbb{N}$ such that $f_{k-1}+1 \leq m \leq f_{k}-2, i \leq n$, $n-i \equiv_{k} f_{k-1}$ for some $i, k \in \mathbb{Z}$ with $k \geq 2$ and $m+i=f_{k}$, we have

$$
\begin{aligned}
& H_{n, m}=\chi(k: 1,2,4) \chi(k: 0,1,2)^{i}(-1)^{[i / 2]} f_{k-2}, \\
& \bar{H}_{n, m}=\chi(k: 2) \chi(k: 3,4,5)^{i}(-1)^{[i / 2]} f_{k-3} .
\end{aligned}
$$

Proof. By the same arguments and in the same notations as in the second part of the proof of Lemma 18, we obtain

$$
\begin{aligned}
& H_{n, m}=\operatorname{det}\left(A_{f_{k-1}+i}^{f_{k}-i} \ldots A_{f_{k}-1}^{f_{k}-i} A_{f_{k}}^{f_{k}-i} \ldots A_{f_{k+1}-1}^{f_{k}-i}\right)
\end{aligned}
$$

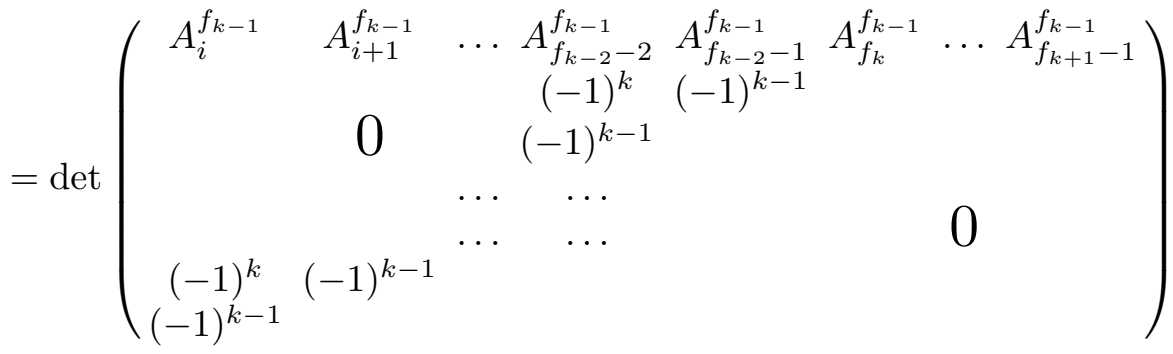

$$
\begin{aligned}
& =(-1)^{(k-1)\left(f_{k-2}-i\right)}(-1)^{\left(f_{k-2}-i\right) f_{k-1}+\left[\left(f_{k-2}-i\right) / 2\right]} H_{f_{k}, f_{k-1}} \\
& =\chi(k: 1,2,4) \chi(k: 0,1,2)^{i}(-1)^{[i / 2]} f_{k-2} \text {. }
\end{aligned}
$$

LEMma 20. For any $n, m \in \mathbb{N}$ such that $f_{k-1}+1 \leq m \leq f_{k}-2, i \leq n$ and $n-i \equiv_{k+1} 0$ for some $i, k \in \mathbb{Z}$ with $k \geq 2$ and $m+i=f_{k}-1$, we have

$$
\begin{aligned}
& H_{n, m}=\chi(k: 0,4) \chi(k: 3,4,5)^{i}(-1)^{[i / 2]} f_{k-2}, \\
& \bar{H}_{n, m}=\chi(k: 2,3,4,5) \chi(k: 0,1,2)^{i}(-1)^{[i / 2]} f_{k-3} .
\end{aligned}
$$


Proof. The proof is similar to the first part of the proof of Lemma 18. With the notation in $(20)$, we get

$$
\begin{aligned}
& H_{n, m}=\operatorname{det}\left(\begin{array}{ccccccc}
A_{i} A_{i+1} & \ldots & A_{f_{k-1}+i-1} & 0 & 0 & \ldots & 0 \\
& & & & & & (-1)^{k} \\
& 0 & & & \ldots & \ldots & \\
& & & (-1)^{k} & (-1)^{k-1} & & 0
\end{array}\right) \\
& =(-1)^{k\left(f_{k-2}-1-i\right)}(-1)^{\left[\left(f_{k-2}-1-i\right) / 2\right]} \operatorname{det}\left(A_{i} A_{i+1} \ldots A_{f_{k-1}+i-1}\right) \text {. }
\end{aligned}
$$

Hence, by Theorem 3

$$
H_{n, m}=\chi(k: 0,4) \chi(k: 3,4,5)^{i}(-1)^{[i / 2]} f_{k-2} .
$$

Lemma 21. For any $n, m \in \mathbb{N}$ such that $f_{k-2}+1 \leq m \leq f_{k}-2, i \leq n$ and $n-i \equiv_{k} f_{k-1}$ for some $i, k \in \mathbb{Z}$ with $k \geq 2$ and $m+i=f_{k}-1$, we have

$$
\begin{aligned}
& H_{n, m}=\chi(k: 2,3,4,5) \chi(k: 0,1,2)^{i}(-1)^{[i / 2]} f_{k-3}, \\
& \bar{H}_{n, m}=\chi(k: 0,4) \chi(k: 3,4,5)^{i}(-1)^{[i / 2]} f_{k-4} .
\end{aligned}
$$

Proof. Since $i=f_{k}-1-m$, we get $1 \leq i \leq f_{k-1}-2$.

If $i=f_{k-2}-1$, then $m=f_{k-1}$ and $n \equiv_{k} f_{k}-1$. Therefore, by Theorem 3 , we get

$$
H_{n, m}=\chi(k-1: 1,2,4) f_{k-3},
$$

which coincides with the required identity since

$$
\begin{gathered}
\chi(k: 0,1,2)^{f_{k-2}-1}=\chi(k:\{0,1,2\} \cap\{0,3\})=\chi(k: 0), \\
(-1)^{\left[\left(f_{k-2}-1\right) / 2\right]}=\chi(k: 0,4) .
\end{gathered}
$$

If $i=f_{k-2}$, then $m=f_{k-1}-1$ and $n \equiv_{k} 0$. Therefore, by Theorem 4 , we get

$$
H_{n, m}=\chi(k-1: 0,4) f_{k-3},
$$

which coincides with the required statement since

$$
\begin{gathered}
\chi(k: 0,1,2)^{f_{k-2}}=\chi(k:\{0,1,2\} \cap\{1,2,4,5\})=\chi(k: 1,2), \\
(-1)^{\left[f_{k-2} / 2\right]}=\chi(k: 3,4) .
\end{gathered}
$$

If $f_{k-2}+1 \leq i \leq f_{k-1}-2$, then $n-i^{\prime} \equiv_{k} 0$ with $i^{\prime}:=i-f_{k-2}$. Then, since $m+i^{\prime}=f_{k-1}-1$ and $f_{k-2}+1 \leq m \leq f_{k-1}-2$, applying Lemma 20, we obtain

$$
\begin{aligned}
H_{n, m} & =\chi(k-1: 0,4) \chi(k-1: 3,4,5)^{i^{\prime}}(-1)^{\left[i^{\prime} / 2\right]} f_{k-3} \\
& =\chi(k: 1,5) \chi(k: 0,4,5)^{i} \chi(k:\{0,4,5\} \cap\{1,2,4,5\})(-1)^{\left[i^{\prime} / 2\right]} f_{k-3} \\
& =\chi(k: 1,4) \chi(k: 0,4,5)^{i}(-1)^{[i / 2]}(-1)^{\left[\left(f_{k-2}+1\right) / 2\right]}(-1)^{i f_{k-2}} f_{k-3} \\
& =\chi(k: 2,3,4,5) \chi(0,1,2)^{i}(-1)^{[i / 2]} f_{k-3} .
\end{aligned}
$$


Now, we consider the case $1 \leq i \leq f_{k-2}-2$. Then, with the notations in (24) and in (20), we get

$$
\begin{aligned}
& H_{n, m}=\operatorname{det}\left(A_{f_{k-1}+i}^{f_{k}-i} \ldots A_{f_{k}-1}^{f_{k}-i} A_{f_{k}}^{f_{k}-i} \ldots A_{f_{k+1}-2}^{f_{k}-i}\right)
\end{aligned}
$$

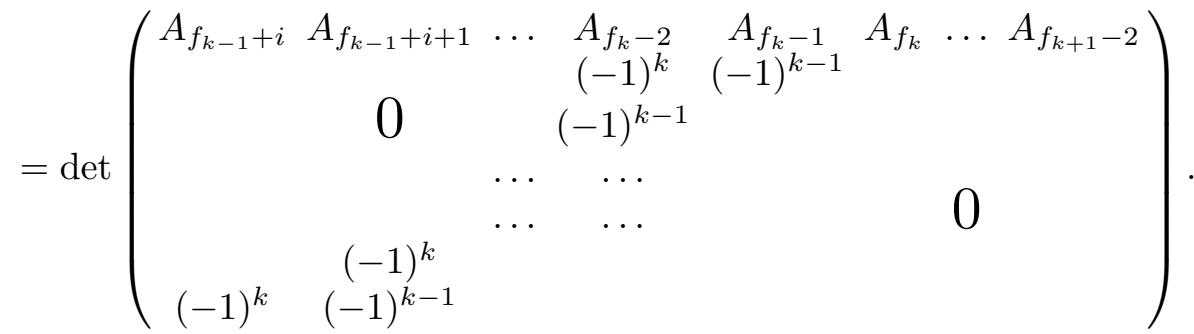

Therefore, by arguments similar to those used in the first part of the proof of Lemma 17, we get

$$
\begin{aligned}
H_{n, m}= & (-1)^{k\left(f_{k-2}-1-i\right)}(-1)^{f_{k-1}\left(f_{k-2}-1-i\right)+\left[\left(f_{k-2}-1-i\right) / 2\right]} \\
& \times\left\{\operatorname{det}\left(A_{f_{k}-1} A_{f_{k}} \ldots A_{f_{k+1}-2}\right)+(-1)^{k-1} \operatorname{det}\left(A_{f_{k}}^{\prime \prime} \ldots A_{f_{k+1}-2}^{\prime \prime}\right)\right. \\
& \left.+(-1)^{k+f_{k-2}-1-i} \operatorname{det}\left(A_{f_{k}}^{\prime \prime \prime} \ldots A_{f_{k+1}-2}^{\prime \prime \prime}\right)\right\},
\end{aligned}
$$

where we use the same notations as in the proof of Lemma 17 except for $A_{j}^{\prime \prime \prime}$ 's which are defined by

$$
A_{j}^{\prime \prime \prime}={ }^{t}\left(\varepsilon_{j} \ldots \varepsilon_{j+f_{k-2}-i-2} \varepsilon_{j+f_{k-2}-i} \ldots \varepsilon_{j+f_{k-1}-1}\right) .
$$

Then, following the arguments there, we get

$$
\begin{aligned}
H_{n, m}= & \chi(k: 4) \chi(k: 0,1,2)^{i}(-1)^{[i / 2]}\left\{H_{f_{k}-1, f_{k-1}}\right. \\
& \left.+(-1)^{k-1} H_{f_{k}+1, f_{k-1}-1}+(-1)^{k+f_{k-2}-1-i} E\right\}
\end{aligned}
$$

with

$$
\begin{aligned}
E & :=\operatorname{det}\left(A_{f_{k}}^{\prime \prime \prime} \ldots A_{f_{k+1}-2}^{\prime \prime \prime}\right) \\
& =\operatorname{det}\left(A_{f_{k}}^{\prime} \ldots A_{f_{k}+f_{k-2}-i-2}^{\prime} A_{f_{k}+f_{k-2}-i}^{\prime} \ldots A_{f_{k+1}-1}^{\prime}\right) \\
& =\operatorname{det}\left(A_{f_{k+1}}^{\prime} \ldots A_{f_{k+1}+f_{k-2}-i-2}^{\prime} A_{f_{k}+f_{k-2}-i}^{\prime} \ldots A_{f_{k+1}-1}^{\prime}\right) \\
& =(-1)^{\left(f_{k-2}-i-1\right)\left(f_{k-3}+i\right)} \operatorname{det}\left(A_{f_{k}+f_{k-2}-i}^{\prime} \ldots A_{f_{k+1}+f_{k-2}-i-2}^{\prime}\right) \\
& =(-1)^{\left(f_{k-2}-i-1\right)\left(f_{k-3}+i\right)} H_{f_{k-2}-i, f_{k-1}-1},
\end{aligned}
$$

where we have used Lemma 5 . Therefore, by Theorems 3 and 4, we have

$$
\begin{aligned}
H_{n, m}= & \chi(k: 4) \chi(k: 0,1,2)^{i}(-1)^{[i / 2]}\left\{\chi(k-1: 1,2,4) f_{k-3}\right. \\
& +(-1)^{k-1} \chi(k-1: 2,3,4,5) f_{k-4} \\
& +(-1)^{k+f_{k-2}-1-i}(-1)^{\left(f_{k-2}-i-1\right)\left(f_{k-3}+i\right)} \\
& \left.\times \chi(k-1: 1,2,3,5) \chi(k-1: 1,4)^{f_{k-2}-i} f_{k-4}\right\} \\
= & \chi(k: 2,3,4,5) \chi(k: 0,1,2)^{i}(-1)^{[i / 2]} f_{k-3} .
\end{aligned}
$$


4. Tiling for $H_{n, m}$ and $\bar{H}_{n, m}$. In this section, we collect the values of $H_{n, m}$ and $\bar{H}_{n, m}$ obtained in the last section and arrange them in the quarter plane $\Omega:=\{0,1, \ldots\} \times\{1,2, \ldots\}$. We will tile $\Omega$ by the following tiles on which the values $H_{n, m}$ are written in. That is, $U_{1}:=V_{1}:=\{(1,-1)\}$, and for $k \geq 2$,

$$
\begin{aligned}
& U_{k}:=\left\{(i, j) \in \mathbb{Z}^{2}: 0 \leq i+j \leq f_{k-1}-1,-f_{k-1} \leq j \leq-1\right\}, \\
& V_{k}:=\left\{(i, j) \in \mathbb{Z}^{2}: 0 \leq i+j \leq f_{k-2}-1,-f_{k-2} \leq j \leq-1\right\},
\end{aligned}
$$

with the written-in values $u_{k}: U_{k} \rightarrow \mathbb{Z}$ and $v_{k}: V_{k} \rightarrow \mathbb{Z}$ given by $u_{1}(1,-1)$ $:=0, v_{1}(1,-1):=1$, and for $k \geq 2$,

$$
\begin{aligned}
& u_{k}(i, j):= \begin{cases}\chi(k: 2) \chi(k: 3,4,5)^{i}(-1)^{[i / 2]} f_{k-3} & (i+j=0), \\
\chi(k: 0,3,4) \chi(k: 0,3)^{i} f_{k-3} & \left(j=-f_{k-1}\right), \\
\chi(k: 3,5) \chi(k: 2,3,4)^{i}(-1)^{[i / 2]} f_{k-3} & \left(i+j=f_{k-1}-1\right), \\
\chi(k: 1,2,3,5) \chi(k: 1,4)^{i} f_{k-3} & (j=-1), \\
0 & (\text { otherwise }),\end{cases} \\
& v_{k}(i, j):= \begin{cases}\chi(k: 1,2,4) \chi(k: 0,1,2)^{i}(-1)^{[i / 2]} f_{k-2} & (i+j=0), \\
\chi(k: 2,3,5) \chi(k: 2,5)^{i} f_{k-2} & \left(j=-f_{k-2}\right), \\
\chi(k: 0,1,2,3) \chi(k: 1,2,3)^{i}(-1)^{[i / 2]} f_{k-2} & \left(i+j=f_{k-2}-1\right), \\
\chi(k: 0,1) \chi(k: 1,4)^{i} f_{k-2} & (j=-1), \\
0 & \text { (otherwise), }\end{cases}
\end{aligned}
$$

and with $\bar{u}_{k}: U_{k} \rightarrow \mathbb{Z}$ and $\bar{v}_{k}: V_{k} \rightarrow \mathbb{Z}$ given $\bar{u}_{1}(1,-1):=1, \bar{v}_{1}(1,-1):=0$, and for $k \geq 2$,

$$
\begin{aligned}
& \bar{u}_{k}(i, j):= \begin{cases}\chi(k: 1,4) \chi(k: 0,1,2)^{i}(-1)^{[i / 2]} f_{k-4} & (i+j=0), \\
\chi(k: 4) \chi(k: 0,3)^{i} f_{k-4} & \left(j=-f_{k-1}\right), \\
\chi(k: 1,2,3,4) \chi(k: 0,1,5)^{i}(-1)^{[i / 2]} f_{k-4} & \left(i+j=f_{k-1}-1\right), \\
\chi(k: 0,1) \chi(k: 1,4)^{i} f_{k-4} & (j=-1), \\
0 & (\text { otherwise), }\end{cases} \\
& \bar{v}_{k}(i, j):= \begin{cases}\chi(k: 2) \chi(k: 3,4,5)^{i}(-1)^{[i / 2]} f_{k-3} & (i+j=0), \\
\chi(k: 3) \chi(k: 2,5)^{i} f_{k-3} & \left(j=-f_{k-2}\right), \\
\chi(k: 2,4) \chi(k: 0,4,5)^{i}(-1)^{[i / 2]} f_{k-3} & \left(i+j=f_{k-2}-1\right), \\
\chi(k: 1,2,3,5) \chi(k: 1,4)^{i} f_{k-3} & (j=-1), \\
0 & \text { (otherwise) } .\end{cases}
\end{aligned}
$$

For $k \geq 1$ let

$$
\begin{aligned}
& \mathcal{U}_{k}:=\left\{\left(n, f_{k}\right): n \in \mathbb{N} \text { and } n \equiv_{k+1} 0\right\}, \\
& \mathcal{V}_{k}:=\left\{\left(n, f_{k}\right): n \in \mathbb{N} \text { and } n \equiv_{k+2} f_{k+1}+f_{k-1}\right\}, \\
& T_{k}:=\left(V_{k}+\left(-f_{k-2}, f_{k}\right)\right) \cap \Omega,
\end{aligned}
$$

where $V+(x, y):=\{(v+x, w+y):(v, w) \in V\}$ for $V \subset \mathbb{Z}^{2},(x, y) \in \mathbb{Z}^{2}$. 
THEOREM 5. We have

$$
\Omega=\bigcup_{k=1}^{\infty}\left(\bigcup_{(i, j) \in \mathcal{U}_{k}}\left(U_{k}+(i, j)\right) \cup \bigcup_{(i, j) \in \mathcal{V}_{k}}\left(V_{k}+(i, j)\right) \cup T_{k}\right),
$$

where the right hand side is a disjoint union, so that $\Omega$ is tiled by the $U_{k}$ 's, $V_{k}$ 's and $T_{k}$ 's. Moreover, for any $(n, m) \in \Omega$, if $(n, m)=(i, j)+\left(i^{\prime}, j^{\prime}\right)$ with $(i, j) \in U_{k}$ and $\left(i^{\prime}, j^{\prime}\right) \in \mathcal{U}_{k}$, then $H_{n, m}=u_{k}(i, j)$ and $\bar{H}_{n, m}=\bar{u}_{k}(i, j)$. Also, if $(n, m)=(i, j)+\left(i^{\prime}, j^{\prime}\right)$ with $(i, j) \in V_{k}$ and either $\left(i^{\prime}, j^{\prime}\right) \in \mathcal{V}_{k}$ or $\left(i^{\prime}, j^{\prime}\right)=$ $\left(-f_{k-2}, f_{k}\right)$, then $H_{n, m}=v_{k}(i, j)$ and $\bar{H}_{n, m}=\bar{v}_{k}(i, j)$. Furthermore, in this tiling, the tiles $U_{k}, V_{k}$ and $T_{k}$ with $k \geq 2$ are followed by the sequences of smaller tiles $U_{k-1} V_{k-1} U_{k-1}, U_{k-1}$ and $U_{k-1}$, respectively, as shown in Figure 1.

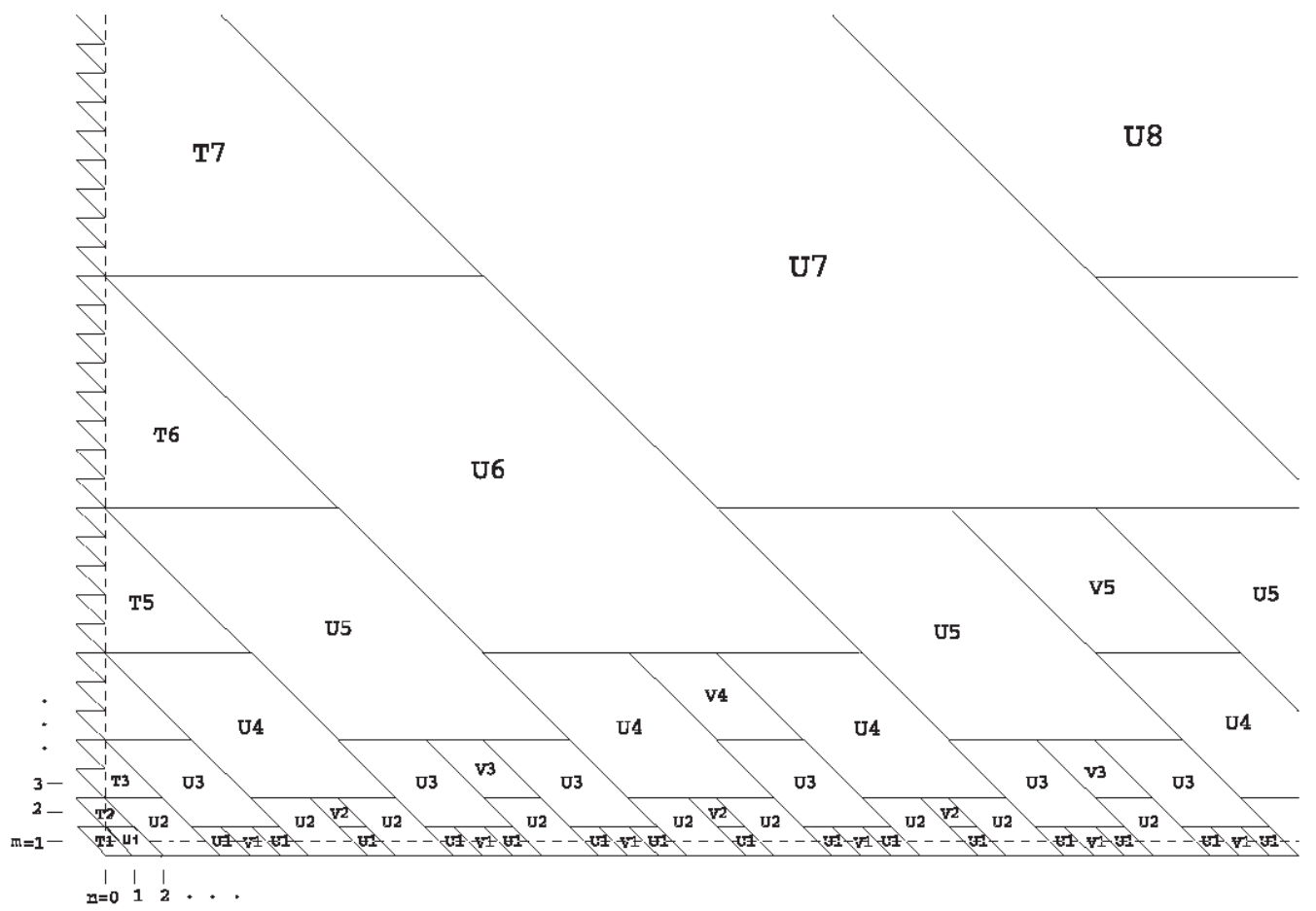

Fig. 1. Tiling for $H_{n, m}$

Proof. Take an arbitrary point $(n, m) \in \Omega$. Let $f_{k-1} \leq m<f_{k}$. If $n+m-f_{k} \geq 0$, define $0 \leq i<f_{k+2}$ by $i \equiv_{k+2} n$.

CASE 1: $n+m-f_{k}<0$. We get $(n, m) \in T_{k}$.

CASE 2: $0 \leq i<f_{k-1}$. We get $(n, m) \in U_{k}+\left(n+m-i-f_{k}, f_{k}\right)$. 
CASE 3: $f_{k-1} \leq i<f_{k+1}$. We get $(n, m) \in U_{k+1}+\left(n+m-i-f_{k+1}, f_{k+1}\right)$.

CASE 4: $f_{k+1} \leq i<f_{k+1}+f_{k-1}$. We get $(n, m) \in U_{k}+(n+m-i+$ $\left.f_{k-1}, f_{k}\right)$.

CASE 5: $f_{k+1}+f_{k-1} \leq i<f_{k+2}$. We get $(n, m) \in V_{k}+(n+m-i+$ $\left.2 f_{k-1}, f_{k}\right)$.

The fact that the written-in values coincide with $H_{n, m}$ and $\bar{H}_{n, m}$ follows from Lemma 18 (first case in $u_{k}$ and $\bar{u}_{k}$ ), Theorem 3 (second case), Lemma 21 (third case), Theorem 4 (fourth case), Corollary 3 (fifth case), Lemma 19 (first case in $v_{k}$ and $\bar{v}_{k}$ ), Theorem 3 (second case), Lemma 20 (third case), Lemma 20 (fourth case) and Corollary 3 (fifth case). The $m$ in the preceding lemmas and theorems coincides with $f_{k}+j$ in Theorem 5 while the meaning of the symbols $k, i, n$ is not necessarily the same.

5. Padé approximation. Let $\varphi=\varphi_{0} \varphi_{1} \ldots$ be an infinite sequence over a field $\mathbb{K}, \widehat{H}_{n, m}:=H_{n, m}(\varphi)$ be the Hankel determinant $(3)$, and $\varphi(z)$ the formal Laurent series (4) with $h=-1$. We also denote the Hankel matrices by

$$
\widehat{M}_{n, m}:=\left(\varphi_{n+i+j}\right)_{i, j=0,1, \ldots, m-1} \quad(n=0,1, \ldots ; m=1,2, \ldots),
$$

so that $\widehat{H}_{n, m}=\operatorname{det} \widehat{M}_{n, m}$.

The following proposition is well known ([1], for example). But we give a proof for self-containment.

Proposition 1. (1) For any $m=1,2, \ldots$, a Padé pair $(P, Q)$ of order $m$ for $\varphi$ exists. Moreover, for each $m$, the rational function $P / Q \in \mathbb{K}(z)$ is determined uniquely for such Padé pairs $(P, Q)$.

(2) For any $m=1,2, \ldots, m$ is a normal index for $\varphi$ if and only if $\widehat{H}_{0, m}(\varphi) \neq 0$.

Proof. Let

$$
\begin{aligned}
& P=p_{0}+p_{1} z+p_{2} z^{2}+\ldots+p_{m} z^{m}, \\
& Q=q_{0}+q_{1} z+q_{2} z^{2}+\ldots+q_{m} z^{m} .
\end{aligned}
$$

Then the condition $\|Q \varphi-P\|<\exp (-m)$ is equivalent to

$$
\begin{aligned}
& -p_{m}=0, \\
& q_{m} \varphi_{0} \quad-p_{m-1}=0, \\
& \text {... } \\
& \begin{array}{rll}
q_{1} \varphi_{0}+\ldots+q_{m} \varphi_{m-1} & -p_{0} & =0 \\
q_{0} \varphi_{0}+\quad \ldots+q_{m} \varphi_{m} & & =0
\end{array} \\
& q_{0} \varphi_{m-1}+{ }_{q_{1}} \varphi_{m-2}+\ldots+q_{m} \varphi_{2 m-1}=0 .
\end{aligned}
$$


Furthermore, the system (26) for $\left(q_{0} q_{1} \ldots q_{m}\right)$ is equivalent to

$$
\left(q_{0} q_{1} \ldots q_{m-1}\right) \widehat{M}_{0, m}+q_{m}\left(\varphi_{m} \varphi_{m+1} \ldots \varphi_{2 m-1}\right)=(00 \ldots 0),
$$

where $\left(p_{0} p_{1} \ldots p_{m}\right)$ is determined by $\left(q_{0} q_{1} \ldots q_{m}\right)$ by the upper half of $(26)$. There are two cases.

CASE 1: $\widehat{H}_{0, m}=0$. In this case, since $\operatorname{det} \widehat{M}_{0, m}=\widehat{H}_{0, m}=0$, there exists a nonzero vector $\left(q_{0} q_{1} \ldots q_{m-1}\right)$ such that $\left(q_{0} q_{1} \ldots q_{m-1}\right) \widehat{M}_{0, m}=0$. Then (27) is satisfied with this $\left(q_{0} q_{1} \ldots q_{m-1}\right)$ and $q_{n}=0$.

CASE 2: $\widehat{H}_{0, m} \neq 0$. In this case, since $\operatorname{det} \widehat{M}_{0, m}=\widehat{H}_{0, m} \neq 0$, there exists a unique vector $\left(q_{0} q_{1} \ldots q_{m-1}\right)$ such that

$$
\left(q_{0} q_{1} \ldots q_{m-1}\right) \widehat{M}_{0, m}=-\left(\varphi_{m} \varphi_{m+1} \ldots \varphi_{2 m-1}\right) .
$$

Then (27) is satisfied with this $\left(q_{0} q_{1} \ldots q_{m-1}\right)$ and $q_{m}=1$.

Thus, a Padé pair of order $m$ exists. Moreover, by the above arguments, a Padé pair $(P, Q)$ of order $m$ with $\operatorname{deg} Q<m$ exists if and only if $\widehat{H}_{0, m}=0$, since if $\widehat{H}_{0, m} \neq 0$, then by $(27), q_{m}=0$ implies $\left(q_{0} q_{1} \ldots q_{m-1}\right)=(00 \ldots 0)$, and hence $Q=0$.

Now we prove that for any Padé pairs $(P, Q)$ and $\left(P^{\prime}, Q^{\prime}\right)$ of order $m$, we have $P / Q=P^{\prime} / Q^{\prime}$. By (5), we have

$$
\|\varphi-P / Q\|<\exp (-n-\operatorname{deg} Q), \quad\left\|\varphi-P^{\prime} / Q^{\prime}\right\|<\exp \left(-m-\operatorname{deg} Q^{\prime}\right) .
$$

Hence,

$$
\left\|P / Q-P^{\prime} / Q^{\prime}\right\|<\exp \left(-m-\operatorname{deg} Q \wedge \operatorname{deg} Q^{\prime}\right)
$$

Therefore,

$$
\left\|P Q^{\prime}-P^{\prime} Q\right\|<\exp \left(-m+\operatorname{deg} Q \vee \operatorname{deg} Q^{\prime}\right) \leq 1 .
$$

Since $P Q^{\prime}-P^{\prime} Q$ is a polynomial of $z,\left\|P Q^{\prime}-P^{\prime} Q\right\|$ is either 0 or not less than 1 . Hence, the above inequality implies $P Q^{\prime}-P^{\prime} Q=0$.

In view of (26), without loss of generality, we can put

$$
\begin{aligned}
& P=p_{0}+p_{1} z+p_{2} z^{2}+\ldots+p_{m-1} z^{m-1}, \\
& Q=q_{0}+q_{1} z+q_{2} z^{2}+\ldots+q_{m} z^{m} .
\end{aligned}
$$

Theorem 6. Let $(P, Q)$ be the normalized Padé pair for $\varphi$ with $\operatorname{deg} Q$ as its normal index $m$ with $P, Q$ given by (29). Then 
(1) $Q(z)=\widehat{H}_{0, m}^{-1} \operatorname{det}\left(z \widehat{M}_{0, m}-\widehat{M}_{1, m}\right)$.

(2) $\operatorname{det}\left(z I-\widehat{M}_{0, m}\right)$ is equal to

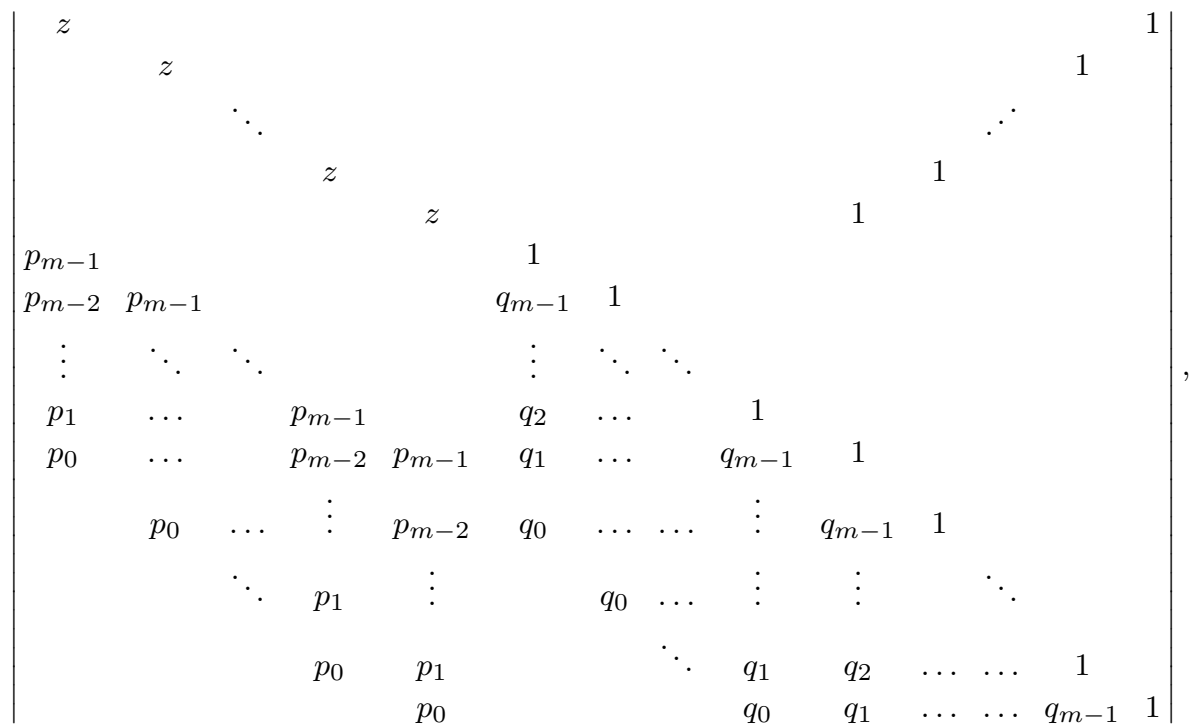

where $I$ is the unit matrix of size $m$.

(3) We have

$$
\widehat{H}_{0, m}=(-1)^{[m / 2]} \prod_{z ; Q(z)=0} P(z)=(-1)^{[m / 2]} p_{k}^{m} \prod_{z ; P(z)=0} Q(z),
$$

where $\prod_{z ; R(z)=0}$ denotes the product over all the roots of the polynomial $R(z)$ with their multiplicity and $p_{k}$ is the leading coefficient of $P(z)$, that is, $p_{m-1}=\ldots=p_{k+1}=0, p_{k} \neq 0$ if $P(z)$ is not the zero polynomial, otherwise $p_{k}=0$.

Proof. (1) Note that $q_{m}=1$ by the assumption that $(P, Q)$ is the normalized Padé pair. By (28), we have

$$
\left(\begin{array}{cccccc}
0 & 1 & & & \\
& 0 & 1 & & & \\
& & & \ddots & & \\
& & & & 0 & 1 \\
-q_{0} & -q_{1} & & \ldots & -q_{m-2} & -q_{m-1}
\end{array}\right) \widehat{M}_{0, m}=\widehat{M}_{1, m} .
$$

Since

$$
\widehat{H}_{0, m}=\operatorname{det} \widehat{M}_{0, m} \neq 0
$$


by the normality of the index $m$, it follows that

$$
\begin{aligned}
& Q(z)=\operatorname{det}\left(z I-\left(\begin{array}{cccccc}
0 & 1 & & & \\
& 0 & 1 & & & \\
& & & \ddots & & \\
& & & 0 & 1 \\
-q_{0} & -q_{1} & & \ldots & -q_{m-2} & -q_{m-1}
\end{array}\right)\right) \\
& =\operatorname{det}\left(z I-\widehat{M}_{1, m} \widehat{M}_{0, m}^{-1}\right) \\
& =\widehat{H}_{0, m}^{-1} \operatorname{det}\left(z \widehat{M}_{0, m}-\widehat{M}_{1, m}\right) \text {. }
\end{aligned}
$$

(2) We define the matrices:

$$
\begin{aligned}
& P_{m}:=\left(\begin{array}{ccccc}
p_{m-1} & p_{m-2} & \ldots & p_{1} & p_{0} \\
p_{m-2} & \ldots & \ldots & p_{0} & \\
\vdots & & . & & \\
p_{1} & . \cdot & & 0 & \\
p_{0} & & & &
\end{array}\right), \\
& P_{m-1}^{\prime}:=\left(\begin{array}{ccccc} 
& & & & p_{m-1} \\
0 & & p_{m-1} & p_{m-2} \\
& & . \cdot & \vdots & \vdots \\
& . \cdot & & \vdots & p_{2} \\
p_{m-1} & p_{m-2} & \ldots & p_{2} & p_{1}
\end{array}\right), \\
& Q_{m}:=\left(\begin{array}{ccccc}
1 & & & 0 & \\
q_{m-1} & 1 & & & \\
\vdots & & \ddots & & \\
\vdots & & & \ddots & \\
q_{1} & q_{2} & \ldots & q_{m-1} & 1
\end{array}\right) \text {, }
\end{aligned}
$$

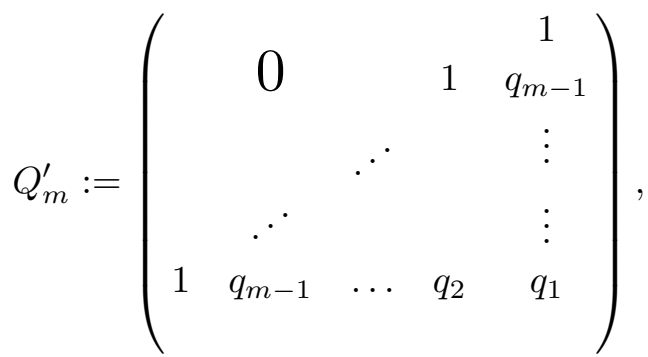




$$
\begin{aligned}
& Q_{m-1}^{\prime \prime}:=\left(\begin{array}{ccccc}
1 & & & 0 & \\
q_{m-1} & 1 & & & \\
\vdots & & \ddots & & \\
\vdots & & & \ddots & \\
q_{2} & q_{3} & \ldots & q_{m-1} & 1
\end{array}\right) \\
& Q_{m, m-1}:=\left(\begin{array}{ccccc}
q_{1} & q_{2} & \ldots & q_{m-2} & q_{m-1} \\
q_{0} & q_{1} & \ldots & q_{m-3} & q_{m-2} \\
& q_{0} & q_{1} & \ldots & q_{m-3} \\
& & \ddots & \ddots & \vdots \\
& 0 & & \ddots & q_{1} \\
& & & & q_{0}
\end{array}\right) \text {, } \\
& \Phi_{m-1}:=\left(\begin{array}{ccccc} 
& & & & \varphi_{0} \\
0 & & \varphi_{0} & \varphi_{1} \\
& & . & \vdots & \vdots \\
& . & & \vdots & \varphi_{m-3} \\
\varphi_{0} & \varphi_{1} & \ldots & \varphi_{m-3} & \varphi_{m-2}
\end{array}\right) .
\end{aligned}
$$

We denote by $O$ the zero matrices of various sizes. We also denote by $I_{n}$ the unit matrix of size $n$. By (26), we have

$$
\begin{aligned}
& \operatorname{det}\left(z I-\widehat{M}_{0, m}\right) \\
& =\operatorname{det}\left(z\left(\begin{array}{cc}
O & O \\
O & I_{m}
\end{array}\right)-\left(\begin{array}{cc}
-I_{m-1} & O \\
Q_{m}^{-1} Q_{m, m-1} & \widehat{M}_{0, m}
\end{array}\right)\right) \\
& =\operatorname{det}\left(\left(\begin{array}{cc}
I_{m-1} & O \\
O & Q_{m}
\end{array}\right)\left(z\left(\begin{array}{cc}
O & O \\
O & I_{m}
\end{array}\right)-\left(\begin{array}{cc}
-I_{m-1} & O \\
Q_{m}^{-1} Q_{m, m-1} & \widehat{M}_{0, m}
\end{array}\right)\right)\right) \\
& =\operatorname{det}\left(z\left(\begin{array}{cc}
O & O \\
O & Q_{m}
\end{array}\right)-\left(\begin{array}{cc}
-I_{m-1} & O \\
Q_{m, m-1} & Q_{m} \widehat{M}_{0, m}
\end{array}\right)\right) \\
& =\operatorname{det}\left(\left(z\left(\begin{array}{cc}
O & O \\
O & Q_{m}
\end{array}\right)-\left(\begin{array}{cc}
-I_{m-1} & \\
Q_{m, m-1} & Q_{m} \widehat{M}_{0, m}
\end{array}\right)\right)\left(\begin{array}{ccc}
I_{m-1} & O & \Phi_{m-1} \\
O & I_{m}
\end{array}\right)\right) \\
& =\operatorname{det}\left(z\left(\begin{array}{cc}
O & O \\
O & Q_{m}
\end{array}\right)-\left(\begin{array}{ccc}
-I_{m-1} & O & -\Phi_{m-1} \\
Q_{m, m-1} & & P_{m}
\end{array}\right)\right) \text {, }
\end{aligned}
$$

where we use (26) to get the last equality. Hence

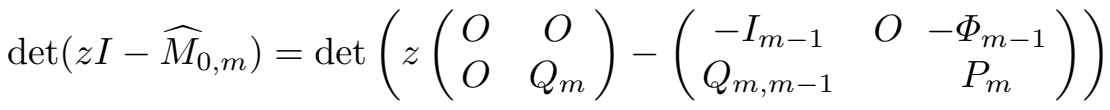




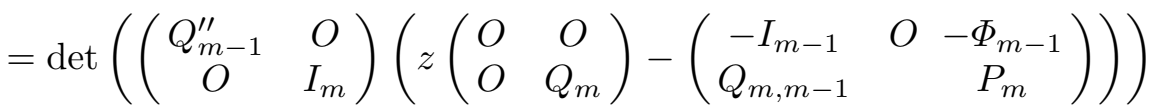

$$
\begin{aligned}
& =\operatorname{det}\left(z\left(\begin{array}{cc}
O & O \\
O & Q_{m}
\end{array}\right)-\left(\begin{array}{ccc}
-Q_{m-1}^{\prime \prime} & O & -P_{m-1}^{\prime} \\
Q_{m, m-1} & P_{m}
\end{array}\right)\right) \\
& =(-1)^{m} \operatorname{det}\left(\left(\begin{array}{ccc}
Q_{m-1}^{\prime \prime} & O & P_{m-1}^{\prime} \\
Q_{m, m-1} & & P_{m}
\end{array}\right)-z\left(\begin{array}{cc}
O & O \\
O & Q_{m}
\end{array}\right)\right) \\
& =(-1)^{m} \operatorname{det}\left(\begin{array}{cccc}
I_{m} & O & & z I_{m} \\
O & Q_{m-1}^{\prime \prime} & O & P_{m-1}^{\prime} \\
Q_{m}^{\prime} & Q_{m, m-1} & & P_{m}
\end{array}\right),
\end{aligned}
$$

which implies (2).

(3) By (2), we have

$$
\begin{aligned}
& \widehat{H}_{0, m}=(-1)^{m} \operatorname{det}\left(0 I-\widehat{M}_{0, m}\right) \\
& =(-1)^{[m / 2]}\left|\begin{array}{ccccccccc}
p_{m-1} & & & & & 1 & & & \\
p_{m-2} & p_{m-1} & & & & q_{m-1} & 1 & & \\
\vdots & & \ddots & & & \vdots & \ddots & \ddots & \\
p_{1} & \ddots & & p_{m-1} & & q_{2} & \ddots & & 1 \\
p_{0} & \ddots & & p_{m-2} & p_{m-1} & q_{1} & \ddots & & q_{m-1} \\
& p_{0} & & & p_{m-2} & q_{0} & \ddots & & q_{m-2} \\
& & \ddots & \vdots & \vdots & & \ddots & & \vdots \\
& & & p_{0} & p_{1} & & & \ddots & q_{1} \\
& & & & p_{0} & & & & q_{0}
\end{array}\right|,
\end{aligned}
$$

which completes the proof since the last determinant is Sylvester's determinant for $P(z)$ and $Q(z)$.

For a finite or infinite sequence $a_{0}(z), a_{1}(z), \ldots$ of elements in $\mathbb{K}\left(\left(z^{-1}\right)\right)$, we use the notation

$$
\left[a_{0}(z) ; a_{1}(z), a_{2}(z), \ldots, a_{n}(z)\right]:=a_{0}(z)+\frac{1}{a_{1}(z)+\frac{1}{a_{2}(z)+}}
$$

and

(30) $\quad\left[a_{0}(z) ; a_{1}(z), a_{2}(z), \ldots\right]:=\lim _{n \rightarrow \infty}\left[a_{0}(z) ; a_{1}(z), a_{2}(z), \ldots, a_{n}(z)\right]$

provided that the limit exists, where the limit is taken with respect to the metric induced by the nonarchimedean norm in $\mathbb{K}\left(\left(z^{-1}\right)\right)$. 
We define

$$
\begin{gathered}
p_{0}(z)=a_{0}(z), \quad p_{-1}(z)=1, \quad q_{0}(z)=1, \quad q_{-1}(z)=0 \\
p_{n}(z)=a_{n}(z) p_{n-1}(z)+p_{n-2}(z), \quad q_{n}(z)=a_{n}(z) q_{n-1}(z)+q_{n-2}(z) \\
(n=1,2,3, \ldots)
\end{gathered}
$$

for any given sequence $a_{1}(z), a_{2}(z), \ldots \in \mathbb{K}\left(\left(z^{-1}\right)\right)$. Then $p_{n}(z), q_{n}(z) \in$ $\mathbb{K}\left(\left(z^{-1}\right)\right), p_{n}(z) \neq 0$ if $q_{n}(z)=0$, and

$$
\frac{p_{n}(z)}{q_{n}(z)}=\left[a_{0}(z) ; a_{1}(z), a_{2}(z), \ldots, a_{n}(z)\right] \in \mathbb{K}\left(\left(z^{-1}\right)\right) \cup\{\infty\} \quad(n \geq 0),
$$

where we mean $\psi / 0:=\infty$ for $\psi \in \mathbb{K}\left(\left(z^{-1}\right)\right) \backslash\{0\}$, and $\psi+\infty:=\infty, \psi / \infty:=0$ for $\psi \in \mathbb{K}\left(\left(z^{-1}\right)\right)$. By using (31), it can be shown that the limit (30) always exists in the set $\mathbb{K}\left(\left(z^{-1}\right)\right)$ as far as

$$
a_{n}(z) \in \mathbb{K}[z] \quad(n \geq 0), \quad \operatorname{deg} a_{n}(z) \geq 1 \quad(n \geq 1) .
$$

For $\varphi(z) \in \mathbb{K}\left(\left(z^{-1}\right)\right)$ given by $(4)$, we denote by $\lfloor\varphi(z)\rfloor$ the polynomial part of $\varphi(z)$, which is defined as follows:

$$
\lfloor\varphi(z)\rfloor:=\sum_{k=0}^{h} \varphi_{h} z^{-k+h} \in \mathbb{K}[z] .
$$

We denote by $T$ the mapping $T: \mathbb{K}\left(\left(z^{-1}\right)\right) \backslash\{0\} \rightarrow \mathbb{K}\left(\left(z^{-1}\right)\right)$ defined by

$$
T(\psi(z)):=\frac{1}{\psi(z)}-\left\lfloor\frac{1}{\psi(z)}\right\rfloor \quad\left(\psi(z) \in \mathbb{K}\left(\left(z^{-1}\right)\right) \backslash\{0\}\right) .
$$

Then, for any given $\varphi(z) \in \mathbb{K}\left(\left(z^{-1}\right)\right)$, we can define the continued fraction expansion of $\varphi(z)$ :

$$
\varphi(z)= \begin{cases}{\left[a_{0}(z) ; a_{1}(z), a_{2}(z), \ldots, a_{N-1}(z)\right]} & \text { if } \varphi(z) \in \mathbb{K}(z), \\ {\left[a_{0}(z) ; a_{1}(z), a_{2}(z), a_{3}(z), \ldots\right]} & \text { otherwise }\end{cases}
$$

with $a_{n}(z)$ satisfying (32) according to the following algorithm.

Continued Fraction Algorithm:

$$
\begin{gathered}
a_{0}(z)=\lfloor\varphi(z)\rfloor, \quad a_{n}(z)=\left\lfloor\frac{1}{T^{n-1}\left(\varphi(z)-a_{0}(z)\right)}\right\rfloor, \\
N=N(\varphi(z)):=\inf \left\{m: T^{m-1}(\varphi(z))=0\right\} \quad(\inf \emptyset:=\infty) .
\end{gathered}
$$

We note that if $\varphi(z) \in \mathbb{K}(z)$, then $N<\infty$; if $\varphi(z) \in \mathbb{K}\left(\left(z^{-1}\right)\right) \backslash \mathbb{K}(z)$, then $N=\infty$ and the continued fraction (33) converges to the given $\varphi(z) \in \mathbb{K}(z)$. We say a continued fraction is admissible if it is obtained by the algorithm. We remark that a continued fraction (33) is admissible if and only if (32) holds.

The following proposition is known [2], but we give a proof for completeness. 
Proposition 2. The set of all $P / Q \in \mathbb{K}(z)$ for Padé pairs $(P, Q)$ for $\varphi(z) \in \mathbb{K}((z))$ coincides with the set of convergents $p_{n}(z) / q_{n}(z)(0 \leq n<N)$ of the continued fraction expansion of $\varphi(z)$. Moreover, $m$ is a normal index if and only if $m$ is a degree of $q_{n}(z)$ for some $n=0,1,2, \ldots$ (with $n<N$ if $\varphi(z) \in \mathbb{K}(z))$.

Proof. Note that

$$
\begin{aligned}
\varphi(z) & =\frac{\left(a_{n}(z)+T^{n}\left(\varphi(z)-a_{0}\right)\right) p_{n-1}(z)+p_{n-2}(z)}{\left(a_{n}(z)+T^{n}\left(\varphi(z)-a_{0}\right)\right) q_{n-1}(z)+q_{n-2}(z)}, \\
(-1)^{n} & =p_{n-1}(z) q_{n-2}(z)-p_{n-2}(z) q_{n-1}(z) .
\end{aligned}
$$

Hence, we have

$$
\begin{aligned}
\left\|q_{n}(z) \varphi(z)-p_{n}(z)\right\| & =\left\|\frac{(-1)^{n} T^{n}\left(\varphi(z)-a_{0}(z)\right)}{q_{n}(z)+T^{n}\left(\varphi(z)-a_{0}(z)\right) q_{n-1}(z)}\right\| \\
& =\exp \left(-\operatorname{deg} a_{n+1}(n)-\operatorname{deg} q_{n}(z)\right)
\end{aligned}
$$

so that

$$
\left\|q_{n}(z) \varphi(z)-p_{n}(z)\right\|<\exp \left(-\operatorname{deg} q_{n}(z)\right) \quad(n<N) .
$$

In the case $N<\infty$, the left-hand side of (34) turns out to be 0 for $n=$ $N-1$. Therefore, $\left(p_{n}(z), q_{n}(z)\right)$ is a Padé pair of order $m=\operatorname{deg} q_{n}(z)$ for all $m \in\left\{\operatorname{deg} q_{n}(z): 0 \leq n<N\right\}$.

Conversely, for any $k=1,2, \ldots$, let $(P, Q)$ be a Padé pair of order $k$. Let $\operatorname{deg} q_{n}(z) \leq k<\operatorname{deg} q_{n+1}(z)$ for some $n=0,1,2, \ldots$ with $n<N$ $\left(\operatorname{deg} q_{N}(z):=\infty\right)$. Then, since $\operatorname{deg} Q \leq k<\operatorname{deg} q_{n+1}$, it follows from (34) that

$$
\begin{aligned}
\left\|\varphi(z)-p_{n}(z) / q_{n}(z)\right\| & =\exp \left(-\operatorname{deg} q_{n}(z)-\operatorname{deg} q_{n+1}(z)\right) \\
& <\exp \left(-\operatorname{deg} q_{n}(z)-\operatorname{deg} Q\right) .
\end{aligned}
$$

Since $(P, Q)$ is a Padé pair of order $k$, we have

$$
\|\varphi(z)-P / Q\|<\exp (-k-\operatorname{deg} Q) \leq \exp \left(-\operatorname{deg} q_{n}(z)-\operatorname{deg} Q\right) .
$$

Therefore,

$$
\left\|\frac{P}{Q}-\frac{p_{n}(z)}{q_{n}(z)}\right\|<\exp \left(-\operatorname{deg} q_{n}(z)-\operatorname{deg} Q\right) .
$$

On the other hand, if $P / Q \neq p_{n}(z) / q_{n}(z)$, then

$$
\begin{aligned}
\left\|\frac{P}{Q}-\frac{p_{n}(z)}{q_{n}(z)}\right\| & =\left\|\frac{P q_{n}(z)-Q p_{n}(z)}{Q q_{n}(z)}\right\| \\
& \geq \exp \left(-\operatorname{deg} q_{n}(z)-\operatorname{deg} Q\right),
\end{aligned}
$$

which is a contradiction. Thus $P / Q=p_{n}(z) / q_{n}(z)$.

Note that $p_{n}(z) / q_{n}(z)$ is irreducible for any $n=1,2, \ldots$ with $n<N$, since $p_{n} q_{n-1}-p_{n-1} q_{n}=(-1)^{n-1}$. Let $m=\operatorname{deg} q_{n}(z)$ for some $n=1,2, \ldots$ with $n<N$. Take any Padé pair $(P, Q)$ of order $m$. Then $\operatorname{deg} Q \leq m$. On the 
other hand, by the above argument, $P / Q=p_{n}(z) / q_{n}(z)$. Since $p_{n}(z) / q_{n}(z)$ is irreducible, this implies that $\operatorname{deg} Q \geq \operatorname{deg} q_{n}(z)=m$. Thus, $m$ is a normal index.

Conversely, let $m \geq 0$ be any normal index. Take any Padé pair $(P, Q)$ of order $m$. Then, by the above argument, there exists $n=0,1,2, \ldots$ with $n<N$ such that $P / Q=p_{n}(z) / q_{n}(z)$. Hence the irreducibility of $p_{n}(z) / q_{n}(z)$ implies $\operatorname{deg} q_{n}(z) \leq \operatorname{deg} Q(\leq m)$. Hence, $\left(p_{n}(z), q_{n}(z)\right)$ is a Padé pair of order $m$. Since $m$ is a normal index, $\operatorname{deg} q_{n}(z)=m$.

We now obtain the continued fraction expansions for

$$
\varphi_{\widehat{\varepsilon}}(z)=\widehat{\varepsilon}_{0} z^{-1}+\widehat{\varepsilon}_{1} z^{-2}+\widehat{\varepsilon}_{2} z^{-3}+\ldots \in \mathbb{Q}\left(\left(z^{-1}\right)\right)
$$

corresponding to the Fibonacci words $\widehat{\varepsilon}=\varepsilon(a, b)$ with $(a, b)=(1,0)$ and $(a, b)=(0,1)$. As in Section 3, we use the notations $\varepsilon$ and $\bar{\varepsilon}$ for them. The proofs in the following theorems are given only for $\varepsilon$, since the proof is similar for $\bar{\varepsilon}$. In [3], J. Tamura gave the Jacobi-Perron-Parusnikov expansion for a vector consisting of Laurent series with coefficients given by certain substitutions, which contains the following as its special case (cf. the footnote on p. 301 of [3]):

Proposition 3. We have

$$
(z-1) \varphi_{\varepsilon}(z)=\left[0 ; z^{f_{-2}}, z^{f_{-1}}, z^{f_{0}}, z^{f_{1}}, z^{f_{2}}, \ldots\right] .
$$

THEOREM 7. We have the following admissible continued fraction for $\varphi_{\varepsilon}(z)$ and $\varphi_{\bar{\varepsilon}}(z)$ :

$$
\varphi_{\varepsilon}(z)=\left[0 ; a_{1}, a_{2}, a_{3}, \ldots\right], \quad \varphi_{\bar{\varepsilon}}(z)=\left[0 ; \bar{a}_{1}, \bar{a}_{2}, \bar{a}_{3}, \ldots\right]
$$

with

$$
\begin{aligned}
& a_{1}=z, \quad a_{2}=-z+1, \quad a_{3}=-\frac{1}{2}(z+1), \\
& a_{2 n+2}=(-1)^{n-1} f_{n}^{2}\left(z^{f_{n}-1}+z^{f_{n}-2}+\ldots+1\right), \\
& a_{2 n+3}=(-1)^{n-1} \frac{1}{f_{n} f_{n+1}}(z-1) \quad(n=1,2, \ldots),
\end{aligned}
$$

and

$$
\begin{aligned}
& \bar{a}_{1}=z^{2}, \quad \bar{a}_{2}=-z, \\
& \bar{a}_{2 n+1}=(-1)^{n-1} f_{n-1}^{2}\left(z^{f_{n}-1}+z^{f_{n}-2}+\ldots+1\right), \\
& \bar{a}_{2 n+2}=(-1)^{n-1} \frac{1}{f_{n-1} f_{n}}(z-1) \quad(n=1,2, \ldots) .
\end{aligned}
$$

Proof. We put

$$
\begin{aligned}
& \theta_{n}:=\left[0 ; z^{f_{n}}, z^{f_{n+1}}, z^{f_{n+2}}, \ldots\right] \quad(n \geq-2), \\
& \xi_{n}:=(-1)^{n-1} \frac{f_{n}^{2} z^{f_{n}}+f_{n-1} f_{n}+f_{n}^{2} \theta_{n+1}}{z-1} \quad(n \geq 1),
\end{aligned}
$$




$$
\begin{aligned}
\eta_{n} & :=(-1)^{n-1} \frac{z-1}{f_{n} f_{n+1}+f_{n}^{2} \theta_{n+1}} \quad(n \geq 1), \\
c_{n} & :=(-1)^{n-1} f_{n}^{2}\left(z^{f_{n}-1}+z^{f_{n}-2}+\ldots+1\right) \quad(n \geq 1), \\
d_{n} & :=(-1)^{n-1} \frac{1}{f_{n} f_{n+1}}(z-1) \quad(n \geq 1) .
\end{aligned}
$$

Then

$$
\xi_{n}=\left[c_{n} ; \eta_{n}\right]\left(=c_{n}+1 / \eta_{n}\right), \quad \eta_{n}=\left[d_{n} ; \xi_{n}\right] .
$$

Using

and Proposition 3, we get

$$
\theta_{n}^{-1}=z^{f_{n}}+\theta_{n+1}
$$

$$
\begin{aligned}
\varphi_{\varepsilon}(z) & =\frac{\theta_{-2}}{z-1} \quad\left(\left\|\theta_{-2} /(z-1)\right\|<1\right) \\
& =\left[0 ;(z-1) \theta_{-2}^{-1}\right] \\
& =\left[0 ; z-1+(z-1) \theta_{-1}\right] \quad\left(\left\|-1+(z-1) \theta_{-1}\right\|<1\right) \\
& =\left[0 ; z, \frac{\theta_{-1}^{-1}}{-\theta_{-1}^{-1}+z-1}\right]=\left[0 ; z, \frac{z+\theta_{0}}{-1-\theta_{0}}\right] \\
& =\left[0 ; z,-z+1+\frac{1+(-z+2) \theta_{0}}{-1-\theta_{0}}\right] \quad\left(\left\|\frac{1+(-z+2) \theta_{0}}{-1-\theta_{0}}\right\|<1\right) \\
& =\left[0 ; z,-z+1, \frac{-1-\theta_{0}^{-1}}{-z+2+\theta_{0}^{-1}}\right] \\
& =\left[0 ; z,-z+1, \frac{-z-1-\theta_{1}}{2+\theta_{1}}\right] \\
& =\left[0 ; z,-z+1,-\frac{1}{2}(z+1), \frac{4 \theta_{1}^{-1}+2}{z-1}\right] \\
& =\left[0 ; z,-z+1,-\frac{1}{2}(z+1), \frac{4 z+2+4 \theta_{2}}{z-1}\right] .
\end{aligned}
$$

Hence, we have

$$
f(z)=\left[0 ; z,-z+1,-\frac{1}{2}(z+1), \xi_{1}\right] \quad\left(\left\|\xi_{1}^{-1}\right\|<1\right) .
$$

From (35) and (36), it follows that

$$
\begin{aligned}
f(z) & =\left[0 ; z,-z+1,-\frac{1}{2}(z+1) c_{1}, d_{1}, \ldots, c_{n}, d_{n}, \xi_{n+1}\right] \\
& =\left[0 ; z,-z+1,-\frac{1}{2}(z+1) c_{1}, d_{1}, c_{2}, d_{2}, \ldots\right]
\end{aligned}
$$

which completes the proof for $\varphi_{\varepsilon}(z)$.

Starting from the identity $\varphi_{\bar{\varepsilon}}(z)=\left(1-\theta_{-2}\right) /(z-1)$ instead of $\varphi_{\varepsilon}(z)=$ $\theta_{-2} /(z-1)$, we can get the admissible continued fraction for $\varphi_{\bar{\varepsilon}}(z)$ in a similar fashion. 
THeOREM 8. The numerator $p_{n}:=p_{n}(z)\left(\bar{p}_{n}:=\bar{p}_{n}(z)\right.$, resp. $)$ and the denominator $q_{n}:=q_{n}(z)\left(\bar{q}_{n}:=\bar{q}_{n}(z)\right.$, resp. $)$ of the $n$th convergent of the continued fraction expansion for $\varphi_{\varepsilon}(z)$ (and $\varphi_{\bar{\varepsilon}}(z)$, resp.) are given as follows:

$$
\begin{aligned}
p_{0}=0, \quad p_{1}=1, \quad p_{2}=-z+1, & \\
q_{0}=1, \quad q_{1}=z, \quad q_{2}=-z^{2}+z+1, & \\
p_{2 n-1}= & \frac{1}{f_{n-1}}\left(\varepsilon_{0} z^{f_{n}-1}+\varepsilon_{1} z^{f_{n}-2}+\ldots+\varepsilon_{f_{n}-1}\right), \\
p_{2 n}= & (-1)^{n}\left\{f_{n-1} z^{f_{n}}\left(\varepsilon_{0} z^{f_{n-1}-1}+\varepsilon_{1} z^{f_{n-1}-2}+\ldots+\varepsilon_{f_{n-1}-1}\right)\right. \\
& \left.-f_{n-2}\left(\varepsilon_{0} z^{f_{n}-1}+\varepsilon_{1} z^{f_{n}-2}+\ldots+\varepsilon_{f_{n}-1}\right)\right\} /(z-1), \\
q_{2 n-1}= & \frac{1}{f_{n-1}}\left(z^{f_{n}}-1\right), \\
q_{2 n}= & (-1)^{n}\left\{f_{n-1} z^{f_{n}}\left(z^{f_{n-1}-1}+z^{f_{n-1}-2}+\ldots+1\right)\right. \\
& \left.-f_{n-2}\left(z^{f_{n}-1}+z^{f_{n}-2}+\ldots+1\right)\right\} \quad(n=2,3, \ldots),
\end{aligned}
$$

and

$$
\begin{aligned}
& \bar{p}_{0}=0, \quad \bar{p}_{1}=1, \\
& \bar{q}_{0}=1, \quad \bar{q}_{1}=z^{2}, \\
& \bar{p}_{2 n-2}=-\frac{1}{f_{n-2}}\left(\bar{\varepsilon}_{0} z^{f_{n}-1}+\bar{\varepsilon}_{1} z^{f_{n}-2}+\ldots+\bar{\varepsilon}_{f_{n}-1}\right), \\
& \bar{p}_{2 n-1}=(-1)^{n-1}\left\{f_{n-2} z^{f_{n}}\left(\bar{\varepsilon}_{0} z^{f_{n-1}-1}+\bar{\varepsilon}_{1} z^{f_{n-1}-2}+\ldots+\bar{\varepsilon}_{f_{n-1}-1}\right)\right. \\
&\left.-f_{n-3}\left(\bar{\varepsilon}_{0} z^{f_{n}-1}+\bar{\varepsilon}_{1} z^{f_{n}-2}+\ldots+\bar{\varepsilon}_{f_{n}-1}\right)\right\} /(z-1)+f_{n-2}, \\
& \bar{q}_{2 n-2}=-\frac{1}{f_{n-2}}\left(z^{f_{n}}-1\right), \\
& \bar{q}_{2 n-1}=(-1)^{n-1}\left\{f_{n-2} z^{f_{n}}\left(z^{f_{n-1}-1}+z^{f_{n-1}-2}+\ldots+1\right)\right. \\
&\left.-f_{n-3}\left(z^{f_{n}-1}+z^{f_{n}-2}+\ldots+1\right)\right\} \quad(n=2,3, \ldots),
\end{aligned}
$$

where $p_{2 n}$ and $\bar{p}_{2 n-1}$ are polynomials since the numerators are divisible by $z-1$.

Proof. The values for $p_{0}, p_{1}, p_{2}, q_{0}, q_{1}, q_{2}$ are obtained from Theorem 7 by direct calculations. For a general $n$, we can prove the formula for $p_{n}, q_{n}$ by induction on $n$ using (31) and Theorem 7 without difficulty.

REMARK 4. From Proposition 2 and Theorem 8, it follows that the set of normal indices for $\varphi_{\varepsilon}(z)$ (and $\varphi_{\bar{\varepsilon}}(z)$, resp.), is $\left\{0, f_{0}=f_{1}-1, f_{1}=f_{2}-\right.$ $\left.1, f_{2}, f_{3}-1, \ldots\right\}\left(\left\{0, f_{1}=f_{2}-1, f_{2}, f_{3}-1, \ldots\right\}\right.$, resp. $)$ which together with Proposition 1 gives another proof of the third cases of Theorem 2 with $n=0$.

REMARK 5. In [4], the continued fraction expansion for Laurent series corresponding to infinite words over $\{a, b\}$ generated by substitutions of "Fibonacci type" is considered, where $a, b$ are viewed as independent variables. 


\section{References}

[1] J.-P. Allouche, J. Peyrière, Z.-X. Wen and Z.-Y. Wen, Some properties of the Hankel determinants associated with the Morse sequence, Ann. Inst. Fourier (Grenoble) 48 (1998), 1-27.

[2] E. M. Nikishin and V. N. Sorokin, Rational Approximations and Orthogonality, Nauka, Moscow, 1988 (in Russian); English transl.: Transl. Math. Monographs 92, Amer. Math. Soc., Providence, R.I., 1991.

[3] J. Tamura, A class of transcendental numbers having explicit g-adic and JacobiPerron expansions of arbitrary dimension, Acta Arith. 71 (1995), 301-329.

[4] - Padé approximation for infinite words generated by certain substitutions, and Hankel determinants, in: Number Theory and Its Applications, K. Győry and S. Kanemitsu (eds.), Kluwer Academic Publishers, to appear.

[5] Z.-X. Wen and Z.-Y. W en, Some properties of the singular words of the Fibonacci word, European J. Combin. 15 (1994), 587-598.

Department of Mathematics

Osaka City University

Osaka, 558-8585 Japan

E-mail: kamae@sci.osaka-cu.ac.jp

Department of Applied Mathematics

Tsinghua University

Beijing 430072, P. R. China

E-mail: wenzy@mail.tsinghua.edu.cn
Faculty of General Education International Junior College

Ekoda 4-5-1, Nakano-ku Tokyo, 165 Japan E-mail: tamura@rkmath.rikkyo.ac.jp 